\title{
THE STUDY OF COMETS AT RADIO WAVELENGTHS
}

\author{
J. Crovisier \\ Observatoire de Paris-Meudon \\ 92195 Meudon FRANCE \\ F. P. Schloerb \\ Department of Physics and Astronomy \\ University of Massachusetts \\ Amherst, MA $01003 \quad$ U.S.A.
}

\begin{abstract}
Radio observations of comets complement studies at other wavelengths as well as providing certain kinds of unique information. Studies of continuum emission may probe the thermal emission of the nucleus and large-size dust particles. Spectroscopic observations of gas in the coma allow searches for parent molecular species that have no signatures in the optical spectral range. The $18-\mathrm{cm}$ wavelength spectral lines of the $\mathrm{OH}$ radical now are relatively easy to detect, and observations of them permit longterm and short-term monitoring of the cometary gaseous output. Moreover, with the unique spectral resolution of radio techniques, aspects of the kinematics of the coma may be also studied, such as the gas expansion velocity and the anisotropy of gas production from the nucleus. In this review, we present recent results of cometary radio observations, and discuss what may be learned from such studies in the future.
\end{abstract}

\section{Introduction}

The history of cometary astronomy has largely been the history of the observation of comets at visible wavelengths. However, with bright comets in the 1970's and 1980's and, most especially, with the 1985-1986 apparition of Halley's Comet, new observational techniques have been exploited to study new aspects of the composition and behavior of comets. As a result, comets in the post-Halley era will be observed throughout the electromagnetic spectrum.

The radio wavelengths occupy many decades of the electromagnetic spectrum from decameter to submillimeter wavelengths, and techniques have been developed which probe all of the major cometary components: the nucleus, the dust, the neutral gas and the plasma. In this respect, radio studies are complementary to other observational techniques since they can provide an independent check on the results obtained at other wavelengths. At the same time, radio data are also a unique source of information in many areas. Radio wavelengths occupy a privileged place in the electromagnetic spectrum: that of the hyperfine lamda-doublet and rotational transitions of most cosmically important molecular species. Thus, radio observations provide a means to observe some cometary molecules that are otherwise difficult to study. Continuum radio observations in the millimeter and microwave parts of the spectrum are sensitive to large dust particles in the coma, and along with radar data (Campbell 1990), provide the best information about this important component of the dust. Finally, certain technical advantages of radio astronomy, such as high spectral resolution, permit physical processes to be investigated that are difficult 
to observe with other techniques.

Radio astronomers have pursued bright comets for over thirty years. Although the first comet for which a detection at radio wavelengths was reported was Comet Arend-Roland 1957 III (c.f. the review of Dobrovolsky 1958), the first radio detection that is now generally accepted is that of the $18-\mathrm{cm} \mathrm{OH}$ transitions in Comet Kohoutek 1973 XII (Biraud et al. 1974; Turner 1974). With modern techniques, most moderately bright comets are detectable in these transitions, and with the observation of $\mathrm{P} / \mathrm{Halley}$, the study of comets at radio wavelengths has entered a new era where long-term coordinated monitoring and sensitive high-frequency observations are now possible (Irvine et al. 1987). The aim of the present paper is to review the results of radio cometary observations and to discuss what may be leamed from such studies in the future. Since several reviews of this topic for pre-Halley observations are available (Snyder 1982, 1986; Crovisier 1985; Schloerb and Gérard 1985), we will only summarize the results obtained during that period, and concentrate on more recent results.

\section{Continuum and Non-Spectroscopic Radio Studies}

\subsection{CONTINUUM OBSERVATIONS}

The study of the continuum radiation from comets has had a confusing history, beginning with reports of emission from Comet Kohoutek (1973 XII). At that time, several groups made observations of the comet at wavelengths close to $3 \mathrm{~cm}$ within several days of each other, and each group reached comparable levels of sensitivity. However, only one group reported a detection at the level of 4 -sigma (Hobbs et al. 1975) with the Green Bank 3-element interferometer, and taken at face value, the observations indicate that the radio emission from the comet must vary on time scales of a day. Observations of Comet West (1976 VI) with the Green Bank interferometer (Hobbs et al. 1977) also yielded both a possible detection on March 5, 1976, at a level of $40 \mathrm{mJy}$, as well as additional evidence for possible day-to-day variation since an upper limit of $15 \mathrm{mJy}$ was achieved on March 4.

Early attempts to explain the centimeter-wave continuum emission that was observed in Comets Kohoutek and West suggested that this emission might arise from large icy grains in the coma, and Gibson and Hobbs (1981) attempted to reconcile the observations with theoretical predictions based upon the Icy Grain Halo model of Delsemme and Miller (1971). However, the predictions of this model fell well below the observed levels, and with the coming of observations on the Very Large Array (VLA), it became clear that these early observations were, at the very least, unusual events. No comet has been detected in the continuum at the VLA, despite search levels that reach two orders of magnitude or more below the reported detections in Comets Kohoutek and West. The levels now reached at the VLA are also well below the predictions of the simple Icy Grain Halo model of Gibson and Hobbs (1981), and it now appears that this particular model has been ruled out by the observations (Snyder et al. 1983). Finally, an assessment of the mass of icy material required to produce the observed level of emission is such that the implied mass production rate is far too large to be a steady state pheneomenon. Thus, if these initial cometary detections are correct, then they are probably related to major outbursts of material from the nucleus, such as occurred in the breakup of Comet West.

In addition to showing the special nature of early radio detections of comets, improved sensitivity at centimeter and millimeter wavelengths has also begun to produce additional detections of comets. In Comet IRAS-Araki-Alcock (1983 VII), the comet was probably detected on May 11 and 12 at $1.3-\mathrm{cm}$ wavelength using the Bonn 100-m antenna (Altenhoff et al. 1983). The signal appeared to be consistent between the two days of observation at a level of $9.0 \pm 0.7 \mathrm{mJy}$. At the same time, other useful observations were taking place that help to constrain possible models of the continuum emission. Observations at 2- and 6-cm wavelengths were carried out at the VLA by de Pater et al. (1985). These failed to detect the comet, but placed interesting upper limits on the flux density of $750 \mu \mathrm{Jy}$ at $2 \mathrm{~cm}$ and 90 $\mu \mathrm{Jy}$ at $6 \mathrm{~cm}$. Perhaps most interesting of all is the fact that radar detections of both the comet nucleus and 
of a cloud of large (centimeter-sized) particles were made at nearly the same time as these radio continuum observations (Goldstein et al. 1984; Harmon et al. 1989). The diameter of the nucleus derived from the radar detection plausibly accounts for the radio emission at $1.3 \mathrm{~cm}$ (de Pater et al. 1985; Harmon et al. 1989). However, the VLA upper limit at $2 \mathrm{~cm}$ is a factor of 4 below the expected blackbody spectrum of the nucleus. Thus, alternative suggestions for the origin of the emission have been made.

Walmsley (1985) has suggested that if the source is extended compared with the VLA synthesized beam, then the observations could be brought into agreement with the expected thermal spectrum of emission from large particles in the coma. Thus, he proposed that the emission arises from meter-sized "boulders" in a 100-km-sized halo surrounding the nucleus. However, analysis of the spectrum of the radar echo by Harmon et al. (1989) suggests that such a halo of boulders would produce a larger radar cross-section than that observed. An alternative explanation to account for the lack of $2-\mathrm{cm}$ emission was suggested by de Pater et al. (1985). They suggested that the brightness temperature of the nucleus could be substantially depressed by subsurface scattering of the radio radiation in the nucleus. Observations of snow and ice fields on the Earth typically show brightness temperatures far below their physical temperature, and if the subsurface scattering properties change enough between $1.3-$ and $2.0-\mathrm{cm}$ wavelengths, then it is conceivable that this mechanism can reconcile the observations. Indeed, the radar echo is consistent with a rough surface that is reminiscent of the Galilean satellites, which are also observed to have unusual surface emissivities at radio wavelengths. More observations of comets at centimeter and millimeter wavelengths are clearly needed to confirm this result.

Continuum observations have also been carried out for $\mathrm{P} / \mathrm{Halley}$. Using the $30 \mathrm{~m}$ telescope of the Institut de Radio Astronomie Millimétrique (IRAM), Altenhoff et al. (1986) detected the comet at a level of $6 \mathrm{mJy}$ at $3.5-\mathrm{mm}$ wavelength and $52 \mathrm{mJy}$ at $1.3-\mathrm{mm}$ wavelength in November 1985 . Later in the apparition, during late March 1986, this group also made repeated and convincing detections of the comet at $1.2-\mathrm{mm}$ wavelength using an extremely sensitive bolometer at the $30-\mathrm{m}$ telescope (Altenhoff et al. 1989). Returning to the November 1985 time period, we note that the comet was also observed at $2 \mathrm{~cm}$ at the VLA by Hoban and Baum (1987), who failed to make a detection at the level of $0.1 \mathrm{mJy}$. Once again, these observations are consistent with the millimeter-wavelength observations of Altenhoff et al. only if the spectrum is steeper than a thermal spectrum.

Consideration of the radio observations of P/Halley in November 1985 and the radar detection made in the same time period suggests that the radio continuum in this comet arises from a halo of large particles surrounding the nucleus rather than from the nucleus itself (Campbell et al. 1989). The known size of the nucleus of Halley cannot account for the observed millimeter-wave emission, nor can it account for the strength and spectral shape of the radar echo. However, calculations of the expected continuum emission from the particles observed by the radar suggest that the observed millimeter-wave emission is consistent with such a model (Campbell et al. 1989), and the lack of an observed 2-cm emission may plausibly be attributed to the cutoff in the particle size distribution at centimeter-sized particles.

Recent continuum results appear to have quantitatively plausible explanations that suggest that emission directly from the nucleus or from large particles in the coma is observable at millimeter wavelengths. Centimeter-wavelength observations, though unsuccessful in these objects, nevertheless play an important role in constraining the possible models of the emission. The amount of radio continuum emission from a cloud of particles is strongly dependent upon the composition of the particles. Thus, continuation of continuum observations of comets should lead to an improved understanding of these large particles, especially if they can be done in conjunction with radar observations, and we note that recent detections of P/Brorsen-Metcalf (1989o) at $1.3-\mathrm{mm}$ wavelength by Altenhoff at the $30-\mathrm{m}$ telescope (private communication) and at $0.8 \mathrm{~mm}$ by Jewitt and Luu (1989) at the James Clerk Maxwell telescope have been quite encouraging. Finally, we note that reports of unusually large radio fluxes have been made for some previous comets and for $\mathrm{P} / \mathrm{Halley}$ as well (Falchi et al. 1987; Scalise et al. 1987). If real, these observations would require unusually large outbursts of material from the nucleus when interpreted in terms of the models that have been discussed above. 


\subsection{OCCULTATION STUDIES}

Occultations of background radio sources by comets offer the potential to make sensitive studies of comets. At low frequencies, the propagation of radio waves through the coma is affected by the plasma associated with the comet, and observations of scintillations of the signal from the background source, as well as changes in its size and position, could lead to constraints on the plasma density and inhomogeneities. Scintillations have been reported in Comet Kohoutek (1973 VII) (Anathakrishnan et al. 1975), and campaigns to observe these effects in P/Halley were undertaken (Alurkar et al. 1986; Anathakrishnan et al. 1987). Both groups have reported enhanced scintillations for some occultation sources, but they differ markedly in the interpretation of these effects. Alurkar et al. (1986) interpret their observations of scintillations of the source PKS2314+03 during December 1985 as a real detection of plasma inhomogeneities in the ion tail of $\mathrm{P} / \mathrm{Halley}$. Under this interpretation, the root mean square (rms) density fluctuations of electrons were 10,6 , and $3 \mathrm{~cm}^{-3}$ on December 18,19 , and 20 , respectively, and the observed periodicity of the scintillations suggests ion density inhomogeneities with scales on the order of $100 \mathrm{~km}$. On the other hand, Anathakrishnan et al. (1987) are critical of this work and state that, given the difficulty of distinguishing scintillations due to the comet from those due to the solar wind and ionosphere, it is difficult to claim an unambiguous detection of the effect. Clearly, observers must take care to make this distinction if the potential of this technique is to be realized.

Occultation observations at higher frequencies, to look at absorption by molecular species in the coma, might someday be useful to make sensitive searches for species that are difficult to detect directly, or for high spatial resolution observations of the coma in a strong molecular line, such as the $18-\mathrm{cm} \mathrm{OH}$ transitions. Observations such as these are known to have been attempted at the VLA in P/Halley (de Pater et al. 1990) though only marginal results have been obtained to date, and one was made by chance during monitoring of the 18-cm OH transitions in comet Okazaki-Levy-Rudenko (1989s) (Bockelée-Morvan et al. 1989). Conceivably, future work in this area could be quite useful if a sufficiently bright source can be found for the experiment.

\section{Spectroscopic Radio Studies}

Cometary radio astronomy has made its most significant advances in the area of radio spectroscopy. The techniques of interpreting these observations closely parallel those that have been developed to interpret observations at shorter wavelengths. In this section, we summarize the current understanding of the molecular excitation of the transitions that are responsible for the radio emission and review the manner in which the data are used to derive molecular production rates. Finally, we discuss observations of $\mathrm{OH}$ and other molecules, with emphasis on those that were obtained during the $\mathrm{P} /$ Halley apparition.

\subsection{EXCITATION CONDITIONS OF MOLECULAR LINES IN COMETARY ATMOSPHERES}

The excitation of cometary molecules arises from a combination of radiative and collisional effects. However, since the density distribution of cometary atmospheres follows, to first approximation, a $1 / \mathrm{r}^{2}$ law, collisional excitation of molecules is significant only in the inner few thousand kilometers of the coma for comets with molecular production rates of about $10^{29} \mathrm{~s}^{-1}$. Therefore, outside this inner region, molecules are out of thermal equilibrium, and their excitation is governed by radiative processes rather than collisions. The most important radiation field to be considered in molecular excitation is that of the Sun, although, to a lesser extent, radiation scattered and emitted by dust in the coma has also been considered theoretically. However, this latter source is important only in the very inner coma, and may be neglected for Earth-based radio observations that have relatively large fields of view.

Radiative excitation of cometary molecules leads to the well-known fluorescence emission process, observed for more than a century in the visible for radicals $\left(\mathrm{OH}, \mathrm{CN}, \mathrm{CH}, \mathrm{C}_{2} \ldots\right)$ and recently in the 
infrared for parent molecules (fundamental bands of $\mathrm{H}_{2} \mathrm{O}$ and $\mathrm{CO}_{2}$ ). At radio wavelengths, the emission from rotational (or hyperfine) transitions is a natural consequence of the radiative decay following excitations at shorter wavelength. Thus, in order to model the radio emission from a particular molecule, one has to solve the entire excitation scheme of the molecule taking into account the rotational and vibrational levels of the ground state and in some cases of one (or several) excited electronic states.

The theory of radio line emission is only a part of the more general theory of molecular line emission at any wavelength, and observation of radio lines can provide an important consistency check on excitation calculations. A good illustration of this is provided by the excitation of the $\mathrm{OH}$ molecule in comets. The inversion of the ground-state ${ }^{2} \Pi_{3 / 2} \mathrm{~J}=3 / 2 \Lambda$-doublet (which is responsible for the 18 -cm lines) is governed by the excitation of the ${ }^{2} \Sigma$ state and subsequent decay in the near ultraviolet (UV). Models (Despois et al. 1981; Schleicher and A'Hearn 1988) can reproduce with reasonable accuracy the rotational structure of the observed UV bands. At the same time, these models simultaneously predict the interesting behavior of the 18-cm observations, which are observed in emission or absorption depending upon the heliocentric radial velocity of the comet, and radio observations can play an important role in checking the validity of the models. The case for the $\mathrm{OH}$ molecule will be further discussed below (Section 3.3).

For most stable (parent) molecules in the coma, the main excitation process is infrared excitation of their fundamental bands of vibration (Crovisier and Encrenaz 1983). Such species do not have significant electronic excitation because their electronic transitions are predissociative and electronic excitation leads to destruction rather than fluorescence. Thus, these species are best observed via their infrared or radio transitions. To a good approximation, radiative excitation is dominated by one or two vibrational bands. The rotational population then results from the competition between the infrared excitation rate and rotational spontaneous decay (Bockelée-Morvan and Crovisier 1985; Crovisier 1987). After a few fluorescence cycles, a steady state situation is established: "fluorescence equilibrium".

For linear molecules without electronic angular momentum, the rotational distribution at fluorescence equilibrium is completely determined by the ratio of total infrared excitation rate to the spontaneous decay rate of the rotational levels. This is shown in Figure 1. The infrared vibrational excitation rate is typically on the order of a few $10^{-4} \mathrm{~s}^{-1}$ at $1 \mathrm{AU}$. As a result, molecules with large rotational Einstein A coefficients will be concentrated on the very first rotational levcls, as in the case of the HCN molecule. On the other hand, molecules with small rotational Einstein $A^{\prime}$ s, such as $\mathrm{CO}$ and $\mathrm{HC}_{3} \mathrm{~N}$, will be spread over many rotational levels (Crovisier and Le Bourlot 1983; Chin and Weaver 1984). This explains why the $\mathrm{J}=1-0$ transition of the minor species HCN could be detected in comet Halley (Despois et al. 1986; Schloerb et al. 1986, 1987b; Bockelée-Morvan et al. 1987; Winnberg et al. 1987), whereas that of the much more abundant $\mathrm{CO}$ molecule remains undetected at radio wavelengths.

Although fluorescence equilibrium provides the basic description of molecular excitation in the coma, there are other important considerations. First of all, fluorescence equilibrium requires several infrared (or electronic) excitation cycles to be established. Therefore, for short-lived molecules, this may not occur and a more rigorous treatment of molecular excitation is needed. In these cases, one needs to solve for the rotational population evolution as the molecules expand outwards, from local thermodynamic equilibrium (LTE) in the collisional region to non-LTE in the outer coma. Examples for a selection of linear molecules are presented in Crovisier (1987).

It is also necessary to include collisions in excitation models in some circumstances. Unfortunately, modelling of collisional excitation in the coma is uncertain due to our poor knowledge of the collisional cross-sections between water and other molecular species and to a lack of direct information on the kinetic temperature in that region. For many radio observations, these limitations are not severe, since the collisional region in the inner few thousand kilometers of the coma is diluted within the field of view and the assumption of fluorescence equilibrium does not significantly affect the interpretation of the data. However, for observations with high spatial resolution, collisional effects must be considered. Conceivably, such observations of several transitions of the same species would allow us to probe the physical conditions of the inner coma and gain new insights into the collisional processes occurring there.

A final complication in the calculation of molecular excitation may arise from radiative transfer. For strong transitions of abundant species, significant optical depths may occur that will affect the molecular 
excitation through radiative trapping. An important example of this effect occurs in the excitation of the water molecule, whose submillimeter rotational transitions are optically thick (Bockelée-Morvan 1987). However, for most cometary species, radiative trapping is not an important effect.

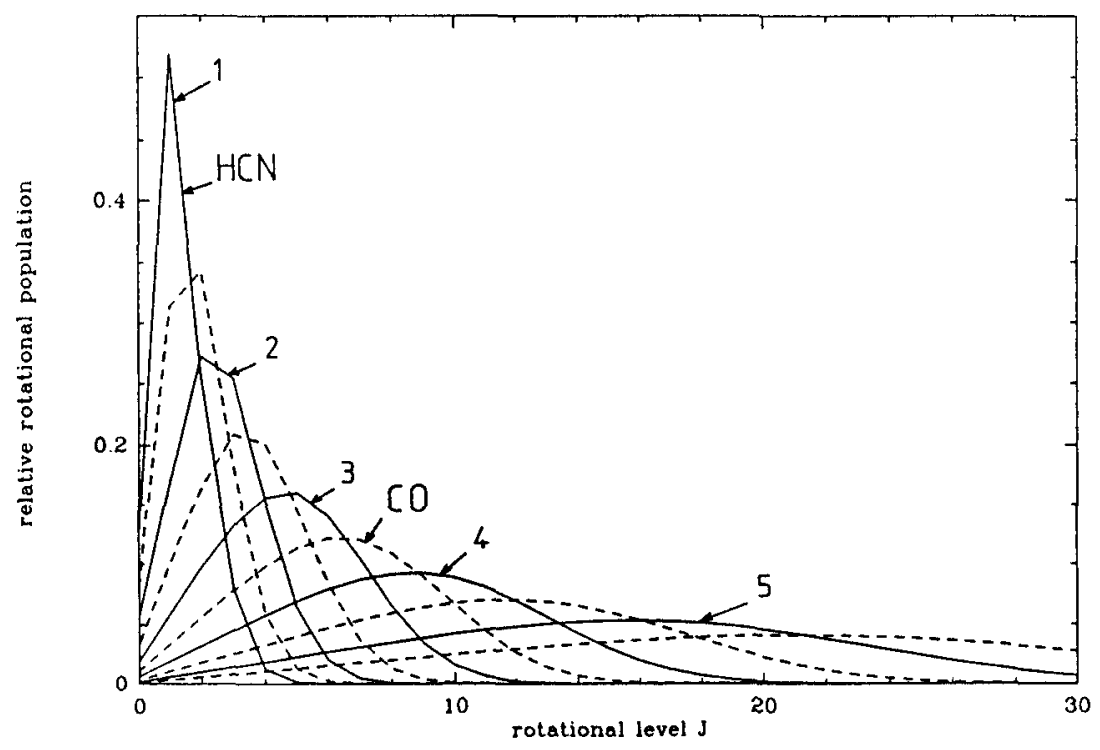

Figure 1. The rotational population distribution of linear molecules at fluorescence equilibrium. The curves display the population distribution as a function of the logarithm of the ratio of the IR excitation rate to the rate of rotational deexcitation from the $\mathrm{J}=1$ level. Data are taken from Table A1 of BockeléeMorvan and Crovisier (1985).

\subsection{DERIVATION OF MOLECULAR ABUNDANCES FROM OBSERVED LINE INTENSITIES}

A fundamental problem of cometary studies is the determination of production rates from the observed spectral line intensities. This problem, which is not trivial and which is the basis of all remote-sensing determinations of molecular abundances, deserves a detailed discussion. The derivation follows a succession of steps, and in this section, we will briefly describe the individual steps in the procedure. In Section 3.3, these steps will be illustrated with a detailed review of the case for the OH radical in comets.

3.2.1. Calibration of Observations. This step is a correction for atmospheric effects, antenna losses, and the coupling between the antenna beam and the source. This latter effect can be easily measured for point (unresolved) sources or for extended sources of uniform brightness. However, determination may be problematic for partially resolved sources such as cometary comae. Calibration can be an important source of difficulties when comparing data obtained with different instruments at different wavelengths.

3.2.2. Determination of Single-State Molecular Column Density in the Beam. This step converts the observed line irtensity into the column density of molecules in the upper (respectively, lower) state of the transition observed in emission (respectively, absorption), averaged over the beam. The conversion is 
trivial when the line is optically thin, which is fortunately the case for the $18-\mathrm{cm}$ lines of $\mathrm{OH}$ and the millimeter and submillimeter lines of the minor species observed or searched for in comets. It is anticipated, however, that the rotational transitions of water between low rotational states are optically thick within several tens of thousands of kilometers of the nucleus for comets with production rates of $10^{29}$ $\mathrm{s}^{-1}$ (Crovisier 1984; Bockelée-Morvan 1988), and the interpretation of such observations would require radiative transfer modelling.

3.2.3. Determination of Total Molecular Column Density in the Beam. The conversion of the number of molecules in a given rotational state into the total number of molecules requires an accurate knowledge of the excitation of this molecule. As discussed in Section 3.1, this step involves a fairly complete treatment of the overall excitation of the molecule in order to find the population of a specific rotational level.

3.2.4. Determination of the Total Number of Molecules in the Coma. For short-lived species directly coming from the nucleus all molecules may be completely included in the beam, and the total number of molecules is directly observed. When this is not the case, the total number could be observed by mapping the entire coma. However, this practice is inconvenient and, for practical purposes, infeasible due to the weakness of the signal. Therefore, one typically has to rely on a model of the molecular density distribution in the coma.

3.2.5. Determination of the Molecular Production Rate. The use of coma models is unavoidable if one wishes to determine the molecular production rate of a comet. Whatever model is adopted (Haser, vectorial, Haser-equivalent), one must know parameters such as molecular scale-lengths, molecular lifetimes, or the expansion velocities of gas in the coma. At the present time, the best information on the velocities may be obtained from the radio line shapes (see Section 4.). Information on scale-lengths may come from mapping (at radio or other wavelengths) or from the comparison of observations obtained with different fields of view (if made simultaneously with coherent calibrations). The lifetimes may also be derived from laboratory measurements or from ab initio calculations.

Any uncertainty (which may mean total lack of information in some cases) in these parameters leads to uncertainty in the production rates. Consequently, it is always good to remember that a particular production rate value is only as good as the model upon which it is based. Indeed, we note that in some cases (e.g., $\mathrm{CO}$ and $\mathrm{H}_{2} \mathrm{CO}$ ), the molecules may be ejected from both the nucleus and from a distributed source in the coma (grains or parent molecules). Clearly, such uncertainties about even the fundamental model to be applied to the data translate into major uncertainties about the production rate determination.

3.2.6. Comparison With Other Species: Relative Abundances. The comparison of abundances of different species requires not only that reliable production rates are determined for these species, but also that they are measured at the same moment, since cometary activity is known to be variable. A further complication is introduced when (as is usually the case) the data for different species come from different instruments, operating in different ranges of wavelengths with different fields of view.

\subsection{STUDIES OF THE OH RADICAL}

The study of the $18-\mathrm{cm}$ wavelength transitions of the $\mathrm{OH}$ radical has been the most productive area of cometary radio astronomy. The $18-\mathrm{cm}$ transitions arise from the ${ }^{2} \Pi_{3 / 2} \mathrm{~J}=3 / 2$ ground state of the $\mathrm{OH}$ radical, which is split into four distinct levels by lambda doubling and hyperfine structure to give rise to the four transitions at 1612, 1665, 1667, and $1721 \mathrm{MHz}$. The first definitive detections of cometary radio emission at any wavelength were made of the $1667-\mathrm{MHz}$ transition of OH in Comet Kohoutek (1974 XII) by Biraud et al. (1974) and Turner (1974). These observations showed that the intensities of the line were quite variable, with the line appearing in emission at some times and in absorption at other times. Biraud et al. (1974) and Mies (1974) provided the basic explanation of this unusual behavior as the result of the Swings effect pumping of the $\mathrm{OH}$ radical by solar UV radiation, the explanation that is generally accepted 
today. Subsequent to the detection of $\mathrm{OH}$ in Comet Kohoutek, these transitions have been sought and detected in many bright comets (see, e.g., Snyder 1986), and a very active campaign of OH observations of P/Halley was undertaken by about a dozen observatories around the world. A summary of these observational programs is presented in Table 1, and sample spectra obtained in December 1985 are shown in Figure 2.

Table 1. P/Halley OH Observing Programs

\begin{tabular}{lrcll}
\hline Telescope & Location & Beam Size(') & Program & Reference \\
\hline NRAO $43 \mathrm{~m}$ & U.S.A. & 18 & Monthly Monitoring & Schloerb et al. (1987a) \\
IAR $30 \mathrm{~m}$ & Argentina & 29 & Daily Monitoring (2/86-5/86) & Bajaja et al. (1987a,b) \\
RATAN 600 & U.S.S.R. & 2 X130 & Single Observations & Bystrova et al. (1987) \\
NRAO VLA & U.S.A. & 1 & High Resolution Mapping & de Pater et al. (1986) \\
CSIRO 64 m & Australia & 13 & Monthly Monitoring & Duncan et al. (1986) \\
DRAO 26 m & Canada & 30 & Daily Monitoring & Galt (1987) \\
Nançay & France & $3.5 X 19$ & Daily Monitoring & Gérard et al. (1987,1988) \\
Arecibo & Puerto Rico & 3 & Monthly Monitoring (8/85-12/85) & Cordes et al. (1986) \\
NRAL 76 m & England & 10 & Daily Monitoring (2/86) & Cohen \\
MPI $100 \mathrm{~m}$ & F.R.G. & 8 & Daily Monitoring (1/86) & Bird et al. (1987) \\
Hartebeestoek & S. Africa & 30 & Daily Monitoring (4/86) & Gaylard (1987) \\
Shanghai $25 \mathrm{~m}$ & China & 30 & Single Observations & Luo et al. $(1988 \mathrm{a}, \mathrm{b})$ \\
Onsala $25 \mathrm{~m}$ & Sweden & 30 & Monthly Monitoring & Winnberg et al \\
\hline
\end{tabular}

* unpublished data

3.3.1. Calibration. Analysis of $\mathrm{OH}$ observations follows the procedure discussed in Section 3.2 above. The first step in the path from observations to production rates is the reduction and calibration of the data themselves. Radio observations at $18 \mathrm{~cm}$ are usually calibrated by comparing the signal from a celestial source with a local noise source coupled to the receiver to account for variations in the system temperature and in the gain of the receiver itself.

The next calibration step is to compare the signal with a celestial source of known flux density. For observations of sources that are small compared with the beam of the radio telescope, this step is straightforward and limited only by the accuracy of the absolute calibration scale in radio astronomy. In this case, observations are generally reported in terms of the flux density of the radio source. For observations of sources that are very large compared with the beam, so that the source may be considered to be uniform in the beam, the signal measured by the observer is more directly related to the intensity of the emission than to the flux, and it is common for observers to report their observations in terms of the "antenna temperature" of the observed emission, which corresponds to the true source brightness temperture in the limiting case of a uniform source that completely fills the antenna beam and all of its sidelobes.

Cometary observations are somewhat problematic, since they represent an intermediate case where the emission region is neither very small nor very large and uniform in the beam. Thus, in the past, cometary $\mathrm{OH}$ results have tended to be reported according to local custom at a particular observatory rather than in a standard form. To facilitate intercomparison between observatories, Schloerb and Gérard (1985) suggested that cometary observations be calibrated and reported as if the comets were point sources in the beam. This procedure has significant advantages over the more commonly used method of reporting antenna temperatures. First of all, it is straightforward to compare the comet signal with known celestial point sources. Thus, calibration is simply defined operationally, and a set of calibrators common to all observers can be defined, whereas calibrations involving local calibration sources are more difficult to intercompare. Moreover, interpretation of antenna temperatures in terms of brightness temperatures requires knowledge of the fraction of power recieved by the antenna that enters through the main antenna beam (the beam efficiency). This quantity is operationally difficult to measure directly, since it requires 
observation of a known uniformly bright source that fills the main telescope beam. On the other hand, the response to a point source, corresponding to a measurement of the gain of the antenna at the beam peak, and the main beam shape are more straightforward quantities to determine. Thus, we suggest that future $\mathrm{OH}$ radio observations follow this suggestion and report their results in terms of the flux density observed in the beam of a particular radio telescope.
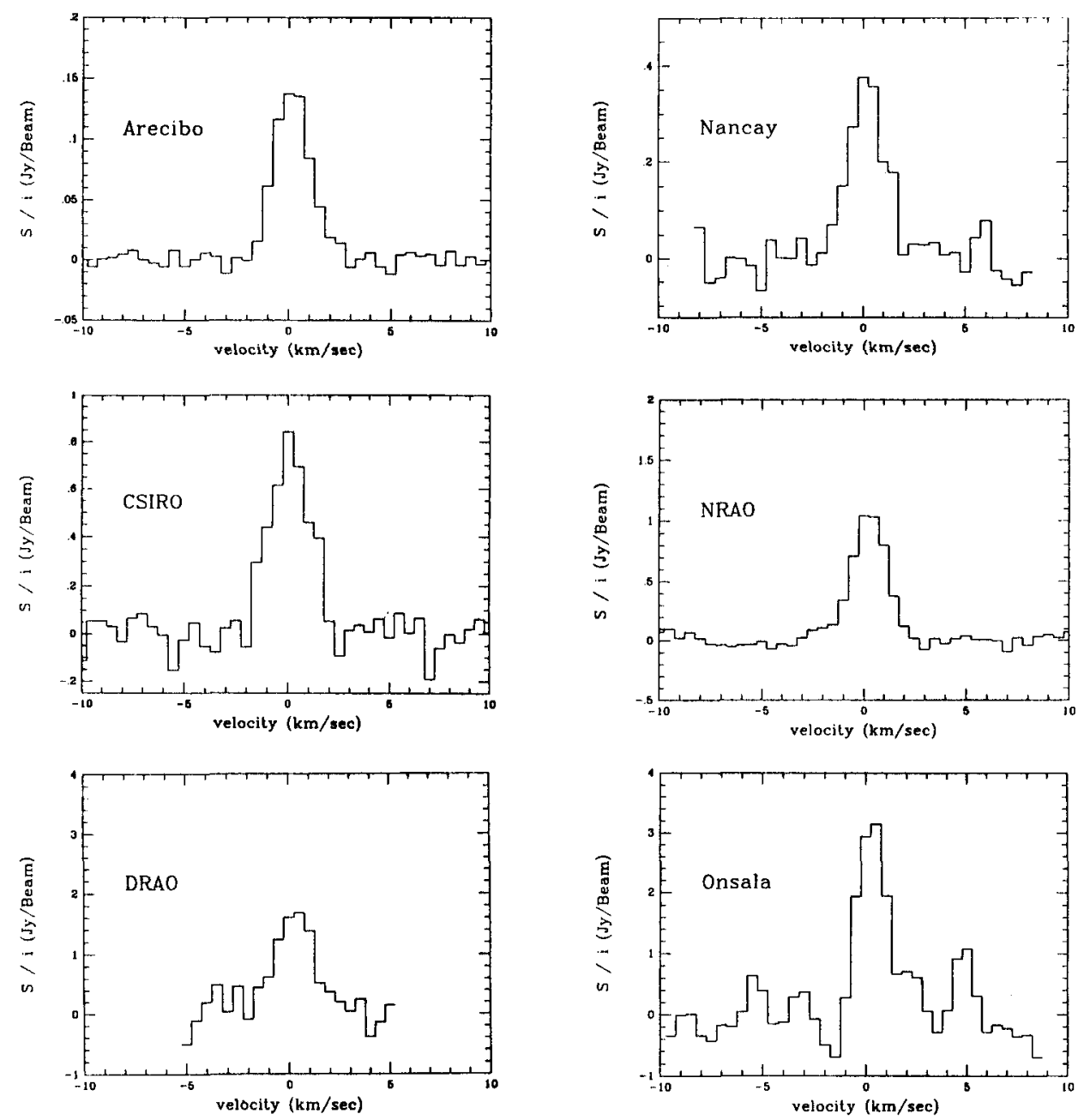

Figure 2. Spectra of $\mathrm{OH}$ in P/Halley obtained during December 1985. Each spectrum is typically an average of several days of observations. Thus, since the $\Lambda$ doublet inversion, $i$, varies during the total time period of the observations, the data in this figure have been normalized by the inversion value that applied at the time of the individual observations before final averaging. All spectra in this figure have been smoothed to a common spectral resolution of $0.5 \mathrm{~km} \mathrm{~s}^{-1}$. 
3.3.2. Analysis. Following the calibration of the data, it is necessary to relate the emission to the number of molecules in the telescope beam. For molecules that are optically thin and radiate by spontaneous emission, it is simple to determine the number of molecules in the upper state of the transition, and to determine the total number of molecules "simply" requires a calculation of the overall excitation. For $\mathrm{OH}$, however, the situation is not so simple, since the $\mathrm{OH}$ emission arises from stimulated emission due to the Swings pumping by solar UV radiation. In this situation, it may be shown (see Schloerb and Gérard 1985) that the flux received is

$$
F=\frac{A_{u l} k T_{b g}}{4 \pi \Delta^{2}} \frac{2 F_{u}+1}{8} \iint B(x, y) \int i(s) n_{O H}(x, y, s) d s d x d y
$$

where $\mathrm{k}$ is Boltzman's constant, $\Delta$ is the Earth-Comet distance, $\mathrm{B}(\mathrm{x}, \mathrm{y})$ is the normalized beam response at position $x, y$ on the sky (i.e., $\iint B(x, y) d x d y=1$ ), $n_{\mathrm{OH}}$ is the number density of $\mathrm{OH}$ in the ground state at position $\mathrm{x}, \mathrm{y}$ and location $\mathrm{s}$ along the line of sight, $\mathrm{T}_{\mathrm{bg}}$ is the brightness temperature of the background emission, $A_{u l}$ is the Einstein A coefficient of the transition, $F_{u}$ is the total angular momentum quantum number of the upper state of the transition, and $i$ is the "inversion" of the lambda doublet, which is defined in terms of the number density of molecules in the upper level $\left(n_{u}\right)$ and lower level $\left(n_{1}\right)$ to be $\mathrm{i}=\left(\mathrm{n}_{\mathrm{u}}-\mathrm{n}_{1}\right) /\left(\mathrm{n}_{\mathrm{u}}+n_{1}\right)$. For illustration, it is easy to assume that the comet is unresolved by the beam and that the inversion i does not vary with position in the coma. Under these conditions, the total flux becomes

$$
\mathrm{F}=\frac{\mathrm{A}_{\mathrm{ul}} \mathrm{kT}_{\mathrm{bg}}}{4 \pi \Delta^{2}} \frac{2 \mathrm{~F}_{\mathrm{u}}+1}{8} \mathrm{i} \mathrm{N}
$$

where $\mathrm{N}$ is now the total number of $\mathrm{OH}$ molecules in the ground state that are present in the coma.

3.3.3. Excitation. The flux in equation 2 above depends linearly upon the population inversion of the ground state lambda-doublet, $\mathrm{i}$, which, in turn, is determined by the Swings effect pumping. As discussed in Section 3.1, the determination of $\mathrm{i}$ is a natural product of models of the overall excitation of the $\mathrm{OH}$ radical. Such models are necessary for the interpretation of both the radio emission and the UV fluorescence of the $\mathrm{OH}$, which offers another important means to monitor the $\mathrm{OH}$ production in comets.

Two models of $\mathrm{OH}$ excitation, by Schleicher and A'Hearn (1988) and by Despois et al. (1981), are commonly used to interpret radio observations. The models are very similar overall, but differ in detail owing to slight differences in the adopted solar spectra. At the present time, neither model is clearly better than the other in explaining the behavior of the $\mathrm{OH}$ radio emission. The calculations of $\mathrm{i}$ in these models, shown in Figure 3, demonstrate that it is a strong function of the heliocentric velocity, since the relative excitation rates of the cometary $\mathrm{OH}$ transitions change as they are Doppler shifted into and out of features in the solar spectrum.

The models of $\mathrm{OH}$ excitation described above assume that the ground state inversion is determined only by the UV excitation process and that no other process is important. However, when the collision rate exceeds the UV excitation rate, the relative populations of the ground-state $\Lambda$ doublet become thermalized and the population inversion, $i$, approaches 0 . Thus, under such circumstances, the radio emission from $\mathrm{OH}$ becomes unobservable, and the emission is said to be "quenched" by the collisions.

Most authors who have investigated $\mathrm{OH}$ excitation in comets, beginning with some very early considerations of the problem (see, e.g., Despois et al. (1981), Elitzur (1981), Schleicher (1983)), have realized that collisions could play an important role in the excitation of the $\mathrm{OH}$ lambda doublet. Recently, Schloerb (1988) has discussed the various possible collision partners and concluded that collisions with 
ions and electrons will be more important than collisions with neutrals, and may influence the effective value of the inversion in the inner coma of comets with high production rates. Collisional quenching is now recognized as an important effect in the calculation of radio emission from comets (Schloerb 1988), and programs are under way to attempt to determine the amount of quenching that occurred in observations of $\mathrm{P} /$ Halley.

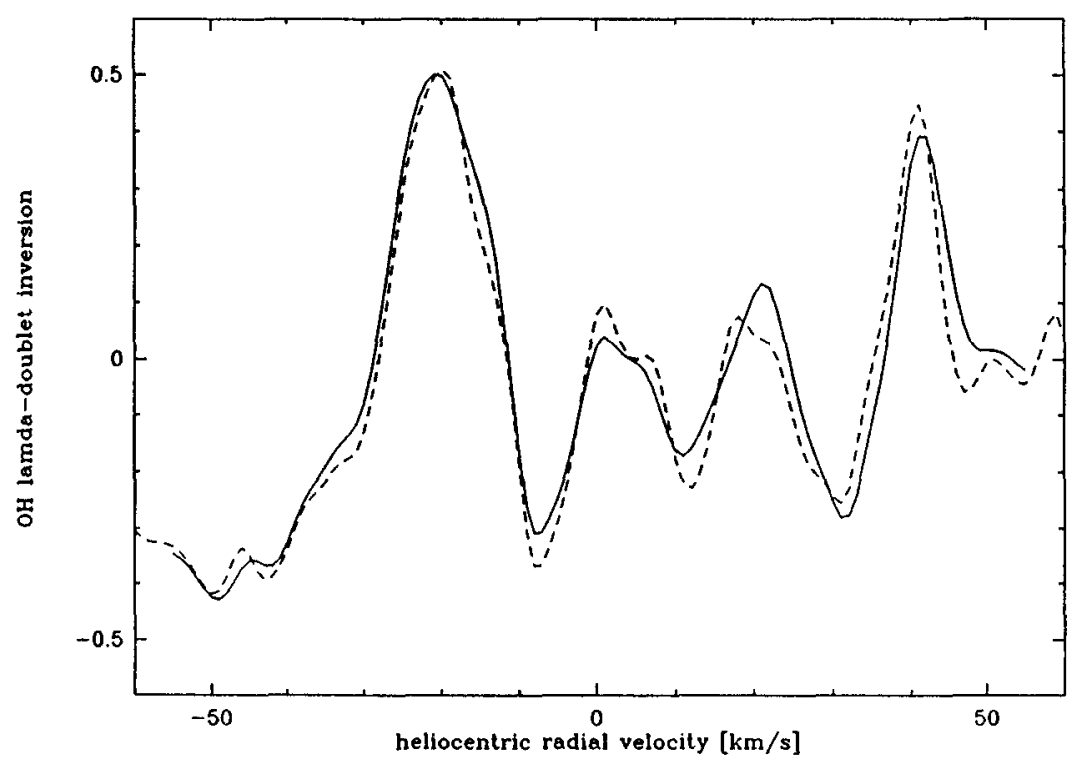

Figure 3. The inversion of the $\mathrm{OH}$ ground state $\Lambda$ doublet, $i$, as a function of heliocentric radial velocity. The solid line shows the model of Despois et al. (1981), and the dashed line shows the model of Schliecher and A'Hearn (1988).

3.3.5. Coma Model. A model of i(s), based upon excitation calculations and including possible adjustments for collisional quenching, is a necessary part of the interpretation of the $\mathrm{OH}$ radio lines. However, it is also apparent from equation 1 that the distribution of molecules with radius in the coma and with location in the main beam is also necessary for the interpretation of the observed flux in terms of the total number of molecules and, eventually, the production rate. To specify this distribution requires a model of the production of $\mathrm{OH}$ from water in the coma and the destruction of $\mathrm{OH}$ due to photodissociation and photoionization. Since such models are necessary for the interpretation of virtually all observations of the coma, they have a long history.

Traditionally, the Haser model (see, e.g., Haser 1957) has been a standard model used for analysis. The Haser model assumes that parent molecules emitted from the nucleus flow radially outward in the coma and that $\mathrm{OH}$ molecules produced by photodissociation of water simply continue this radial outward motion. However, studies of brightness profiles of the coma and of the shapes of radio $\mathrm{OH}$ lines (see Section 4 . below) have indicated that a better description of this process is provided by the vectorial model (Combi and Delsemme 1980; Festou 1981). In this model, the radial distribution of OH is calculated by assuming that the additional velocity taken by the dissociated $\mathrm{OH}$ molecule is randomly directed with respect to the radial direction and added vectorially to the original outward velocity of water. 
Within the vectorial model, it is necessary to specify several parameters describing the photolysis of water and $\mathrm{OH}$ and the kinematics of these molecules. Many models have been suggested previously, based on theoretical estimates of these quantities, and some examples of the traditionally used models are given in Table 2. However, we note that modern coma models, which are an indication of the models to be used in the "post-Halley era", have become more sophisticated than this, allowing for velocity dispersions about the mean value or variations in the parent velocity with production rate or heliocentric distance.

Table 2. Examples of Standard Vectorial Models for the OH Coma

\begin{tabular}{lcccc} 
Model & I & $\begin{array}{c}\text { Traditional Models } \\
\text { II }\end{array}$ & II & Post-Halley Model \\
\hline Reference & $(1)$ & $(2)$ & $(3)$ & This paper \\
$\mathrm{v}_{\mathrm{p}} \mathrm{km} \mathrm{s}^{-1}$ & 0.9 & 1.0 & 0.9 & Variable with $\mathrm{r}_{\mathrm{h}}, \mathrm{Q}_{\mathrm{p}}(4)$ \\
$\tau_{\mathrm{p}} \mathrm{s}$ & $8 \times 10^{4}$ & $8.2 \times 10^{4}$ & $1.0 \times 10^{5}$ & $8.2 \times 10^{4}$ \\
$\mathrm{v}_{\mathrm{OH}} \mathrm{km} \mathrm{s}^{-1}$ & 1.15 & 1.15 & 1.30 & Velocity distribution about 1.1 (5) \\
$\tau_{\text {OH }} \mathrm{s}$ & $1 \times 10^{5}$ & $2 \times 10^{5}$ & $4 \times 10^{5}$ & $1-2 \times 10^{5}(6)$ \\
Quenching of Radio Emission & None & None & Included & Included (7)
\end{tabular}

(1) "Radio Model 1986a"

(2) Festou's Vectorial Model - the coma model used to interpret IUE UV observations

(3) Vectorial model based on work of Despois et al. (1981)

(4) Tacconi-Garman (1989); Bockelée-Morvan et al. (1990); Tacconi-Garman et al. (1990)

(5) Crovisier (1989)

(6) e.g., Van Dishoeck and Dalgamo (1984); Schleicher and A'Heam (1988)

(7) Schloerb (1988)

3.3.6. OH Production. The first effort to utilize a complete model of both $\mathrm{OH}$ excitation and the coma to analyze radio data from many comets was carried out in Despois et al. (1981). This paper analyzed observations of several comets from the late 1970's at the Nançay Radio Observatory and demonstrated many of the important properties of the $\mathrm{OH}$ radio emission. The major result of the paper was an attempt to derive the $\mathrm{OH}$ production rates from radio data alone, including the estimation of parameters of the coma from radio observations. The result of the work was to demonstrate that the radio-derived production rates were correlated with the optical brightness of the comet, and hence with other measures of the gas production. However, another important result was that there were systematic differences between the estimate of $\mathrm{OH}$ production from observations of the UV and radio lines, with the UV-derived value typically exceeding the radio-derived value.

Although some of the systematic differences between published UV and radio-derived $\mathrm{OH}$ production rates may be attributed to the use of different coma models, real physical effects, such as collisional quenching of the radio emission from the inner coma, appear to be important factors. Knowledge of the quenching region in comets has increased considerably as a result of observations of $\mathrm{P} / \mathrm{Halley}$. For one thing, VLA observations of P/Halley by de Pater, Palmer and Snyder (see the companion paper in this book) have probably directly observed the quenched region in the center part of their maps of the $\mathrm{OH}$ emission in the comet. On another front, recent efforts using the mass of data at all wavelengths collected on Halley's Comet have emphasized the simultaneous use of UV and radio data to derive the production rate and constrain physical parameters (including quenching) in coma models. For example, Gérard (1990) has presented an analysis of UV and radio data obtained on P/Halley in December 1985. Since the UV 
Table 3. Spectral Line Searches in P/Halley

\begin{tabular}{|c|c|c|c|}
\hline Transition & Frequency (MHz) & Telescope & Reference \\
\hline $\mathrm{CI}^{3} \mathrm{p}_{1}-{ }^{3} \mathrm{p}_{0}$ & 492162 & $\mathrm{KAO}$ & Keene et al." \\
\hline $\mathrm{C}_{3} \mathrm{H}_{2} 1_{10^{-1}}$ & 18343 & NRAO $43 \mathrm{~m}$ & Matthews et al. ${ }^{*}$ \\
\hline $\mathrm{CH}_{3} \mathrm{CN} 3-2$ & 36392 & Haystack $37 \mathrm{~m}$ & Webber and Haschick" \\
\hline \multirow[t]{2}{*}{$\mathrm{CH}_{3}^{3} \mathrm{CN} 5-4$} & 91959 & IRAM $30 \mathrm{~m}$ & Bockelée-Morvan et al. (1987) \\
\hline & & FCRAO $14 \mathrm{~m}$ & Swade et al. (1987) \\
\hline $\mathrm{CH}_{3} \mathrm{CN} 6-5$ & 110360 & OSO $20 \mathrm{~m}$ & Winnberg et al. (1987) \\
\hline $\mathrm{CH}_{3}^{3} \mathrm{CN} \mathrm{v}_{8}=16-5$ & 110709 & FCRAO $14 \mathrm{~m}$ & Swade et al. (1987) \\
\hline $\mathrm{CH}_{3} \mathrm{OH}_{2}-3-1 \mathrm{E}$ & 12178 & NRAO $43 \mathrm{~m}$ & Batrla* \\
\hline $\mathrm{CH}_{3} \mathrm{OH} 15_{3}-144_{4} \mathrm{~A}+$ & 88590 & OSO $20 \mathrm{~m}$ & Winnberg et al. (1987) \\
\hline $\mathrm{CH}^{2} \Pi_{1 / 2} \mathrm{~J}=1 / 2 \mathrm{~F}=0-1$ & 3264 & NRAO $43 \mathrm{~m}$ & Turner \\
\hline $\mathrm{CH}^{2} \Pi_{1 / 2}^{1 / 2} \mathrm{~J}=1 / 2 \mathrm{~F}=1-1$ & 3335 & $\mathrm{NR} \Lambda O 43 \mathrm{~m}$ & Tumer" \\
\hline $\mathrm{CH}^{2} \Pi_{1 / 2}^{1 / 2} \mathrm{~J}=1 / 2 \mathrm{~F}=2-1$ & 3349 & NRAO $43 \mathrm{~m}$ & Tumer" \\
\hline $\mathrm{CO}^{+} \mathrm{N}=2-1 \mathrm{~J}=5 / 2-3 / 2$ & 236063 & NRAO $12 \mathrm{~m}$ & Baum and Hoban (1986) \\
\hline $\mathrm{CO} 2-1$ & 230538 & IRAM $30 \mathrm{~m}$ & Bockelée-Morvan et al. (1987) \\
\hline HI $21 \mathrm{~cm}$ & 1420 & RATAN 600 & Bystrova et al." \\
\hline \multirow[t]{2}{*}{$\mathrm{H}_{2} \mathrm{CO}_{10}{ }^{-1} 11$} & 4830 & NRAO VLA & Snyder et al. (1989) \\
\hline & & MPI $100 \mathrm{~m}$ & Bird et al. (1987) \\
\hline \multirow{4}{*}{$\begin{array}{l}\mathrm{H}_{2} \mathrm{CO} 2_{11}-2{ }_{12} \\
\mathrm{H}_{2} \mathrm{O} 3_{13}-200 \\
\mathrm{H}_{2} \mathrm{O}_{14} 4_{32}^{-3}\end{array}$} & 14488 & NRAO $43 \mathrm{~m}$ & Batrla* \\
\hline & 183310 & $\mathrm{KAO}$ & Gulkis et al." \\
\hline & 380197 & $\mathrm{KAO}$ & Gulkis et al (1989) \\
\hline & & KAO & Keene et al. \\
\hline \multirow{4}{*}{$\mathrm{H}_{2} \mathrm{O} 6_{16}-523$} & 22235 & MPI $100 \mathrm{~m}$ & Bird et al. (1987) \\
\hline & & IRO $14 \mathrm{~m}$ & Scalise et al. (1987) \\
\hline & & $\mathrm{NRAO} 43 \mathrm{~m}$ & Batrla" \\
\hline & & LPI $22 \mathrm{~m}$ & Berulis et al. (1987) \\
\hline \multirow{2}{*}{$\mathrm{H}_{3} \mathrm{O}^{+} \mathrm{l}_{1}-2$} & 307192 & NRAO $12 \mathrm{~m}$ & Wooten et al. (1986) \\
\hline & & MWO $5 \mathrm{~m}$ & Wootten et al. (1986) \\
\hline \multirow[t]{2}{*}{$\mathrm{HC}_{3} \mathrm{~N} 4-3$} & 36796 & Haystack $37 \mathrm{~m}$ & Webber and Haschick \\
\hline & & LPI $22 \mathrm{~m}$ & Berulis et al. (1987) \\
\hline $\mathrm{HC}_{3} \mathrm{~N} 5-4$ & 45000 & NRO $45 \mathrm{~m}$ & Kaifu et al. \\
\hline $\mathrm{HC}_{3}^{3} \mathrm{~N} 10-9$ & 90971 & IRAM $30 \mathrm{~m}$ & Bockelée-Morvan et al. (1987) \\
\hline $\mathrm{HC}_{3} \mathrm{~N} 11-10$ & 100076 & IRAM $30 \mathrm{~m}$ & Bockelée-Morvan et al. (1987) \\
\hline $\mathrm{HC}_{3} \mathrm{~N} 12-11$ & 109174 & FCRAO $14 \mathrm{~m}$ & Swade et al. (1987) \\
\hline \multirow[t]{5}{*}{$\mathrm{HCN} 1-0$} & 88638 & FCRAO $14 \mathrm{~m}$ & Schloerb et al. (1986) \\
\hline & & IRAM $30 \mathrm{~m}$ & Despois et al. (1986) \\
\hline & & $\operatorname{OSO} 20 \mathrm{~m}$ & Winnberg et al. (1987) \\
\hline & & HCRAO Interferometer & Wright et al." \\
\hline & & $\mathrm{NRO} 45 \mathrm{~m}$ & Kaifu et al." \\
\hline $\mathrm{HCO}^{+} 1-0$ & 89189 & FCRAO $14 \mathrm{~m}$ & Swade ct al. (1987) \\
\hline HNC $1-0$ & 90664 & FCRAO $14 \mathrm{~m}$ & Swade et al. (1987) \\
\hline $\mathrm{NH}_{3} 1_{1}-1$ & 23694 & MPI $100 \mathrm{~m}$ & Bird et al. (1987) \\
\hline \multirow{2}{*}{$\mathrm{NH}_{3} 3_{3}-3$} & 23870 & $\mathrm{NRAO} 43 \mathrm{~m}$ & Batrla" \\
\hline & & NASA DSN (Tidbinbilla) $64 \mathrm{~m}$ & Gulkis et al. ${ }^{*}$ \\
\hline OCS $8-7$ & 97301 & IRAM $30 \mathrm{~m}$ & Bockelée-Morvan et al. (1987) \\
\hline $\mathrm{OH}^{2} \Pi_{1 / 2} \mathrm{~J}=1 / 2 \mathrm{~F}=1-0$ & 4765 & MPI $100 \mathrm{~m}$ & Bird et al. (1987) \\
\hline $\mathrm{OH}^{2} \Pi_{3 / 2}^{1 / 2} \mathrm{~J}=7 / 2 \mathrm{~F}=4-4$ & 13441 & NRAO $43 \mathrm{~m}$ & Batrla* \\
\hline $\mathrm{SiO} v=11-0$ & 43122 & Haystack $37 \mathrm{~m}$ & Webber and Haschick ${ }^{*}$ \\
\hline
\end{tabular}

* unpublished data 
emission is not affected by collisional quenching, maps of the UV brightness were used to constrain the radial distribution of $\mathrm{OH}$. At the same time, radio spectra were used to constrain the velocities in the coma and to derive the scale of the region that suffers from collisional quenching. Gerard found that both radio and UV observations could be shown to be consistent if the radio emission is quenched within $70,000 \mathrm{~km}$ from the nucleus. Utilizing this result for the quenching radius, and scaling the value for the production rate as discussed in Schloerb (1988), Bockelée-Morvan et al. (1990) have shown that the radio OH production rates can be brought into reasonable agreement with UV-derived values when consistent coma parameters are used and quenching is accounted for. However, we note that at the present time many of these results are of a preliminary nature and further work may lead to some refinements in the models.

\subsection{OTHER MOLECULAR SPECIES}

The appearance of Halley's Comet in 1985-1986 provided an important opportunity to make searches for new molecules with radio telescopes and to attempt to confirm the presence of molecules that have possibly been detected in previous comets. Table 3 lists the searches for transitions, other than the 18-cm $\mathrm{OH}$ transitions, that were carried out at radio wavelengths in P/Halley. Hydrogen cyanide (HCN) and formaldehyde $\left(\mathrm{H}_{2} \mathrm{CO}\right)$ were detected. However, the detections of some other species, which have been claimed in the past $\left(\mathrm{CH}_{3} \mathrm{CN}, \mathrm{NH}_{3}\right.$, the $22-\mathrm{GHz}$ line of $\mathrm{H}_{2} \mathrm{O}$ ), were not confirmed. The nondetection of most species in Table 3 is in overall agreement with what we know (or expect) about molecular excitation and cometary molecular abundances. In several cases, however, the derived upper limits on the production rates do put significant constraints on the cometary chemical composition.

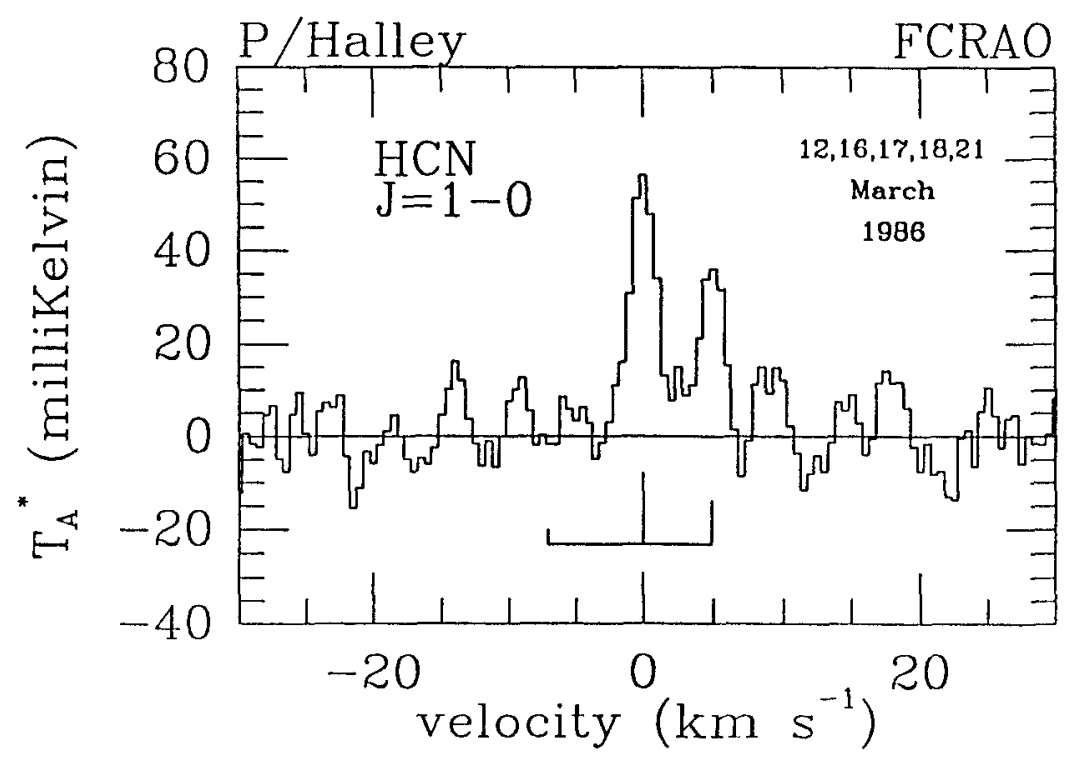

Figure 4. Spectrum of the $\mathrm{J}=1-0$ transition of $\mathrm{HCN}$ in P/Halley, obtained by Schloerb et al. (1987b) at the Five College Radio Astronomy Observatory $14 \mathrm{~m}$ telescope during March 1986. The figure beneath the spectrum indicates the relative positions and strengths of the hyperfine components of this transition. 
Detection of the J=1-0 lines of HCN at $89 \mathrm{GHz}$ in Comet Kohoutek 1973 XII was announced by Huebner et al. (1974). Subsequent searches in other comets with a higher sensitivity were negative, or at most gave marginal results (Irvine et al. 1984; Bockelée-Morvan et al. 1984). However, the presence of $\mathrm{HCN}$ in comets was confirmed when the $\mathrm{HCN}$ radio lines were finally unambiguously detected in $\mathrm{P} / \mathrm{Halley}$ (see, e.g., Figure 4) by three radio telescopes (Despois et al. 1986; Schloerb et al. 1986, 1987b; BockeléeMorvan et al. 1987; Winnberg et al. 1987) and monitored for nearly six months. Recent searches for these lines were negative in Comet Wilson 1987 VII (Crovisier et al. 1990), but probably successful in comet P/Brorsen-Metcalf (1989o) (Colom et al. 1989).

The HCN line intensities observed in $\mathrm{P} / \mathrm{Halley}$ correspond to an $\mathrm{HCN}$ production rate on the order of 0.001 that of water. $\mathrm{HCN}$ is thus a minor constituent of comets that is observable at radio wavelengths because of favorable excitation conditions (see Section 3.1 and Figure 1); its observation in the infrared would be hopeless with presently available techniques. It should be noted that higher rotational transitions of $\mathrm{HCN}$ (especially the J=3-2 at $266 \mathrm{GHz}$ ) are still more favorably excited, and were marginally detected in P/Brorsen-Metcalf (1989o) by Colom et 21. (1989). The HCN production rate compared with that of $\mathrm{CN}$ shows that hydrogen cyanide is a major progenitor of the $\mathrm{CN}$ cometary radical. There are indications, however, that the $\mathrm{HCN}$ production rate might be smaller (by about a factor of two) than the CN production rate in $\mathrm{P} / \mathrm{Halley}$. This fact is also consistent with the HCN upper limits observed in many other comets (Bockelée-Morvan et al. 1984; Crovisier et al. 1989). The notable exception to this trend is the HCN signal observed in Comet Kohoutek (1973 XII). The level of $\mathrm{HCN}$ emission in it was much larger than that observed in $\mathrm{P} / \mathrm{Halley}$ and, when interpreted with excitation models that explain the $\mathrm{P} / \mathrm{Halley}$ data, this observation would require a much larger $\mathrm{HCN}$ abundance than observed in $\mathrm{P} / \mathrm{Halley}$. Since the $\mathrm{CN}$ abundance of Comet Kohoutek was not anomalous, we suggest that this early result might be spurious, although other explanations, such as a different $\mathrm{HCN}$ excitation mechanism or a truly anomalous abundance, are also possible.

Confirmation that $\mathrm{HCN}$ is not the sole parent of $\mathrm{CN}$ will require careful evaluation of the $\mathrm{HCN}$ and $\mathrm{CN}$ density distributions and lifetimes. If this is the case, then additional $\mathrm{CN}$ sources would need to be identified. Searches for some possible $\mathrm{CN}$ parents $\left(\mathrm{HNC}, \mathrm{HC}_{3} \mathrm{~N}_{2} \mathrm{CH}_{3} \mathrm{CN}\right.$ ) were carried out in $\mathrm{P} / \mathrm{Halley}$ with negative results that appear to preclude them as major contributors of $\mathrm{CN}$. Other molecular species have also been postulated, such as $\mathrm{C}_{2} \mathrm{~N}_{2}$, which, unfortunately, has no rotational transitions. It is also possible that the additional $\mathrm{CN}$ may come directly from grains, as suggested by the jet-like $\mathrm{CN}$ distributions observed by A'Hearn et al. (1986).

The $1_{11^{-1}}$ transition of $\mathrm{H}_{2} \mathrm{CO}$ at $5 \mathrm{GHz}$ was detected at the 3.4 sigma level in $\mathrm{P} / \mathrm{Halley}$ and at the 2sigma level in Comet Machholtz 1988 XV by Snyder et al. (1990) using the VLA. Although these detections are marginal in our opinion, their credibility is reinforced by the detection of the $v_{1}-v_{5}$ bands of $\mathrm{H}_{2} \mathrm{CO}$ by the infrared spectrometer aboard the VEGA probes (Combes et al. 1988) and by the Neutral Mass Spectrometer (NMS) observations aboard Giotto (Krankowsky et al. 1990). Both radio and in situ observations lead to a formaldehyde production rate that is a few percent that of water, which suggests that $\mathrm{H}_{2} \mathrm{CO}$ is an important cometary constituent. Moreover, the $31_{12}^{-2}$ transition of $\mathrm{H}_{2} \mathrm{CO}$ at $226 \mathrm{GHz}$ has also been tentatively detected in comet P/Brorsen-Metcalf 1989 o (Colom et al. 1989), and future observations of $\mathrm{H}_{2} \mathrm{CO}$ at the VLA and at millimeter-wavelength telescopes are to be encouraged.

The water molecule deserves special discussion. The detection of the $66_{16}^{-5}$ line at $22 \mathrm{GHz}$, claimed in comets Bradfield 1974 III (Jackson et al. 1976) and IRAS-Araki-Alcock 1983 VII (Altenhoff et al. 1983), was not confirmed in other comets. In fact, it is now known from infrared observations that the water rotational distribution is mostly subthermal (Weaver et al. 1989), as predicted by excitation models (Crovisier 1984; Bockelée-Morvan 1987). The 22-GHz transition occurs between two rotational levels at $643 \mathrm{~K}$ above the zero point energy. Although this transition might be inverted in some regions of the coma, the column densities of the relevant rotational levels are much too small to yield a significant signal. The $414^{-3}$ transition at $380 \mathrm{GHz}$ also occurs between relatively high rotational states; this explains why Gulkis et al. (1989) failed to detect it with the Kuiper Airborne Observatory in P/Halley. In contrast, water rotational transitions between low rotational states are predicted to be very intense. These transitions fall in the submillimeter range and can only be observed from space. The $1_{10^{-1}}$ line at $557 \mathrm{GHz}$, between the 
two lowest rotational states of ortho-water, looks very promising and should be observable even in modest comets by future space submillimeter telescopes.

\section{Kinematic Studies}

\subsection{RADIO SPECTROSCOPY AND COMA KINEMATICS}

High spectral resolution is a unique advantage of radio spectroscopy. Although other techniques applied to cometary spectroscopy (e.g., Fabry-Perot spectroscopy in the visible: Roesler et al. 1986; Fouriertransform spectroscopy in the infrared: Larson et al. 1987) may yield resolutions of up to 200,000, this is insufficient to fully characterize cometary line profiles, which have typical widths of about $2 \mathrm{~km} / \mathrm{s}$, and to fully study the coma velocity field, which has typical velocities of $1 \mathrm{~km} / \mathrm{s}$. The resolution of radio spectroscopy is only limited by the signal-to-noise ratio. Since the molecular lines are optically thin (if one excepts the submillimeter transitions of water) and purely Doppler, measurement of their shape provides us with the gas velocity distribution along the line of sight. This measurement permits two important effects to be studied: the coma expansion velocity, which is related to the line widths, and the nucleus anisotropic outgassing, which is related to the velocity offsets of the lines with respect to the nucleus rest velocity.

\subsection{PROBING GAS EXPANSION AND THERMODYNAMICAL MODELS}

The gas expansion velocity is a basic parameter for the description of the coma and is a requisite for the derivation of constituent abundances from observed emissions. Hydrodynamical models of the coma (c.f. Crifo 1989 and references therein) predict that gas, starting at a velocity close to $0.35 \mathrm{~km} / \mathrm{s}$ in the vicinity of the nucleus surface, is then accelerated through rotational-translational energy conversion and by photolytic heating. However, ill-known additional processes such as gas-dust interaction, radiative cooling and water recondensation must also be taken into account. The gas terminal velocity is expected to depend on both the heliocentric distance (which governs the photolytic rate) and the gas production rate (which governs the collision rate, and therefore the thermalization of the fast photolytic decay products).

Given the complexity of the physical processes involved in the theoretical calculation of the outflow velocity, it has always been critical to have observational data to constrain these models. Before the Halley results, one had to rely largely on very indirect estimations of this parameter, based on the observation of halo expansions, or of the Greenstein effect, and the empirical law $v_{\text {exp }}=0.58 \mathrm{r}_{\mathrm{h}}^{-0.5} \mathrm{~km} \mathrm{~s}^{-1}$ derived by Delsemme (1982) was widely accepted. However, the unique direct determination of the coma expansion velocity that was provided by the in situ measurement of the NMS experiment aboard Giotto (Lämmerzahl et al. 1987) shows that this empirical law does not fully represent the behavior of comets. The NMS experiment found that the neutral gas progressively increased from $0.8 \mathrm{~km} / \mathrm{s}$ at a few thousand kilometers from the nucleus to more than $1.0 \mathrm{~km} / \mathrm{s}$ at $30,000 \mathrm{~km}$. In general, these values are in reasonable agreement with the model predictions (Bockelée-Morvan and Crovisier 1987; Combi and Smyth 1988; Hodges 1990), but they are significantly larger than the predictions of Delsemme's empirical law.

In order to build upon the unique Giotto results, observations of the gas expansion velocity in a variety of comets representing a range of conditions are needed, and radio line shapes provide the best means to study the behavior of the coma expansion velocity on a routine basis. Parent molecule line shapes are directly related to this parameter. The thermal line widths being small for the expected coma kinetic temperatures, the observed line widths should be close to twice the coma expansion velocity. The HCN millimeter lines observed in $\mathrm{P} / \mathrm{Halley}$ (Figure 5) lead to $\mathrm{v}_{\exp }$ ranging from $0.8 \mathrm{~km} / \mathrm{s}$, when the comet was at about $1.5 \mathrm{AU}$ from the Sun, to $1.3 \mathrm{~km} \mathrm{~s}^{-1}$, around perihelion when the gas production rate was higher (Bockelée-Morvan et al. 1987; Schloerb et al. 1987b). These figures are consistent with the NMS/Giotto measurement, with the model predictions, and with the expected variation with $\mathrm{r}_{\mathrm{h}}$ and gas production rate. 


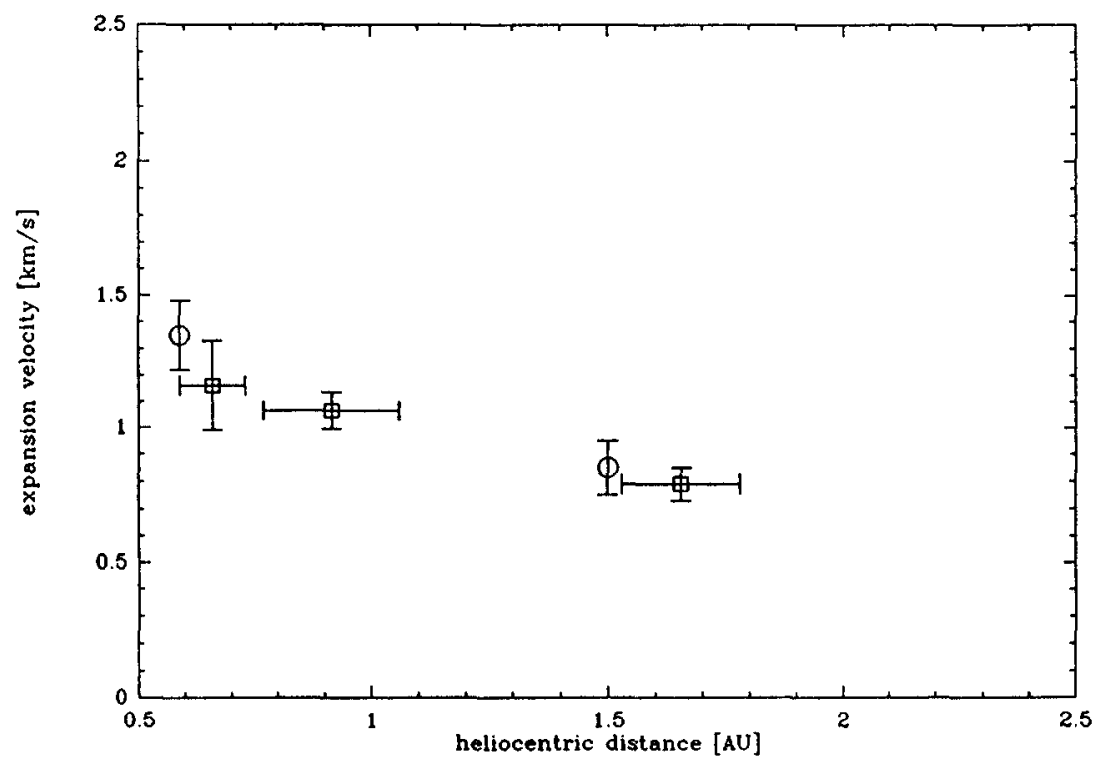

Figure 5. Coma expansion velocity of $\mathrm{P} / \mathrm{Halley}$ as a function of heliocentric distance derived from observations of the HCN line width. Circles represent IRAM data obtained by Bockelée-Morvan et al. (1987); squares represent the FCRAO data of Schloerb et al. (1987b).

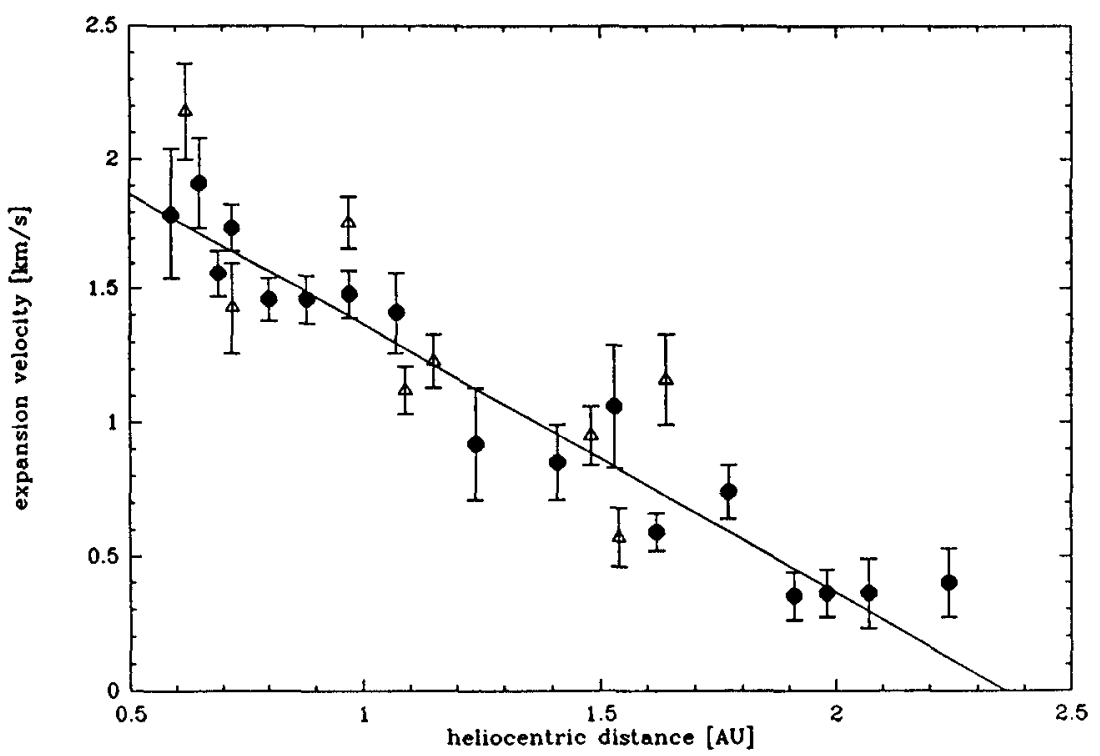

Figure 6. Coma expansion velocity of P/Halley as a function of heliocentric distance derived from the 18$\mathrm{cm}$ OH line widths measured at the Nançay radio telescope (Bockelée-Morvan et al. 1990). Filled circles represent data obtained before perihelion; triangular points show post-perihelion data. 
One can also attempt to retrieve the gas expansion velocity from the radio $\mathrm{OH}$ lines, which have been observed in several comets for a variety of heliocentric distances and gas production rates (Figure 6). In this case, the information on the parent molecule expansion velocity is less direct, because the $\mathrm{OH}$ radical produced from water photolysis is given an additional velocity $v_{\text {eject }}$ which adds isotropically to $v_{\text {exp }}$. One has then to compare observed profiles with synthetic profiles, which can be obtained by Monte Carlo simulation (Bockelée-Morvan and Gérard 1984; Schloerb et al. 1987b; Tacconi-Garman et al. 1990). In order to reduce the number of free parameters, one can adopt a model value for $v_{\text {eject }}$. Crovisier (1989) has shown from recent laboratory measurements that the distribution of $v_{\text {eject }}$ is close to the mean value 1.05 $\mathrm{km} / \mathrm{s}$. It is then possible to deconvolve the $\mathrm{OH}$ velocity profiles to derive $\mathrm{v}_{\exp }$ (Bockelée-Morvan et al. 1990). Such analyses were performed on an important set of observations of $P /$ Halley as well as several other comets, sampling a large range of heliocentric distances and gas production rates. As shown in Figure 6, the resulting $\mathrm{v}_{\text {exp }}$ are found to vary in a range 0.5 to $2.5 \mathrm{~km} / \mathrm{s}$ (i.c., broader than that found for the HCN lines), and to depend, as expected, both on $r_{h}$ and on the gas production rate (Bockelée-Morvan et al. 1990; Tacconi-Garman et al. 1990). It is interesting to note that the smaller values $(0.5 \mathrm{~km} / \mathrm{s})$ consistently found for P/Halley in October 1985 and for less gaseous comets like P/Giacobini-Zinner or Wilson 1987 VII are smaller than expected from standard hydrodynamical models. The reasons for this discrepancy are unclear at the present time.

\subsection{PROBING GAS ANISOTROPY AND NON-GRAVITATIONAL FORCES}

Modelling non-gravitational forces exerted on cometary nuclei is an important and attractive topic of cometary physics. It allows us to understand and predict long-term evolution of cometary orbits. Compared with non-gravitational acceleration parameters determined from astrometric measurements, it also allows us to estimate nucleus masses, and subsequently, nucleus densities. Indeed, it is at present the only method that provides access to these basic parameters (Rickman 1986, 1988; Sagdeev et al. 1988).

Non-gravitational forces are due to the anisotropy of the outgassing from the nucleus, which occurs preferentially on the side heated by the Sun. A net jet force is resulting, which may be written:

$$
\mathrm{F}_{\mathrm{jet}}=-\mathrm{Q} \zeta \mathrm{v}_{\mathrm{i}}
$$

where $Q$ is the gas production rate (in mass), $v_{i}$ is the gas initial velocity, and $\zeta$ is an efficiency factor that depends on the outgassing pattern, namely on the collimation of gas jets (which are between unidirectional and isotropic) and on the distribution of active regions on the nucleus. In their first studies, Rickman (1986) and Sagdeev et al. (1988) tried to evaluate $v_{i}$ and $\zeta$ from theory only (hydrodynamics and thermodynamics). The authors do not agree on that point. $v_{\mathrm{i}}$ is expected to be close to $0.35 \mathrm{~km} / \mathrm{s}$, but there is a large uncertainty on $\zeta$. Furthermore, $F_{\text {jet }}$ may not be directed opposite to the Sun, due to the heating lag if the nucleus is rotating. This lag angle, which depends on the nucleus rotation and on the thermal properties of the nucleus, is a further uncertainty of the model. Due to all these uncertainties, and also to that on the gas production rate and its variation over the whole cometary orbit, there are large uncertainties in cometary mass and density determinations.

The observation of cometary line shapes piovides an elegant way to measure the jet velocities directly : the first moment of the line profile with respect to the nucleus velocity (the "line velocity offset") is just the projection of $\zeta \mathrm{v}_{\mathrm{i}}$ on the line of sight. Velocity offsets were actually measured for the $\mathrm{OH}$ and HCN radio lines in P/Halley: They are indeed directed towards the Sun, with values in the range of $0.0-0.3 \mathrm{~km} / \mathrm{s}$, dcpending on the comet phase angle (Bockelée-Morvan et al. 1987; Schloerb et al. 1987a, b; Colom 1989; Colom et al. 1990). Similar values were also obtained from analysis of the infrared water lines (Larson et al. 1987) and the radio formaldehyde observations of Snyder et al. (1990). A detailed analysis of the OH observations leads to the determination $\zeta \mathrm{v}_{\mathrm{i}}=0.17 \pm 0.02 \mathrm{~km} \mathrm{~s}^{-1}$ and shows that the lag angle due to nucleus rotation is small and positive (Colom 1989; Colom et al. 1990). These facts not only provide a check of hydrodynamical and thermal models at the nucleus surface, they also inform us about the overall pattern of cometary jets. Since radio observations provide both the projection of $\zeta v_{\mathrm{i}}$ and the gas production rate and 
its evolution, a complete modelling of non-gravitational forces may even be attempted from these observations alone (Colom 1989). A significant improvement of the mass and density determinations may be expected.

\section{Cometary Radio Observations in the Post-Halley Era}

The observation of comets at radio wavelengths is now a mature technique that both complements and enhances information obtained by other techniques. Several developments may be expected in the future, as instrumentation improves and as more comets are observed at radio wavelengths. In this section, we discuss the future prospects for radio observations of comets.

\subsection{IMPROVEMENT OF INSTRUMENTATION}

The progress of cometary radio astronomy has largely been due to improvements in radio instrumentation. We expect that this trend will continue in the post-Halley era, and that new instrumentation will provide new opportunities for cometary observers. Large telescopes will be equipped in the near future with even more sensitive receivers, and with arrays of beams that will allow mapping of the weak cometary emissions. Instrumental improvements are expected to be particularly significant at high frequencies, where some important molecular species are expected to have strong transitions and where observations of the continuum emission from large dust grains are more feasible. The submillimeter range, which will become accessible from space telescopes in the next decade, is especially noteworthy in this regard.

\subsection{RADIO CONTINUUM OBSERVATIONS}

The continuum detections of Comet Halley and other recent comets have shown that, with high sensitivity, it is now possible to study a previously unsuspected component of large-size grains in the coma. Several sensitive continuum systems for one-millimeter and submillimeter observations are in operation around the world, and this dust component should soon become observable in a number of comets. Thus, although detections of continuum emission are few in number at the present time, we expect that this area will grow in the near future.

\subsection{RADIO OBSERVATIONS OF OH}

Monitoring the $\mathrm{OH}$ radio lines in comets is a useful (and, being insensitive to solar elongation or weather conditions, often unique) way to monitor the cometary gas output. The various secondary processes that affect the $\mathrm{OH}$ radio line intensities are now well-understood and it appears to be possible to reasonably reconcile production rate determinations from various sources. Systematic radio monitoring of $\mathrm{OH}$ in future comets will be of particular interest to observe both long-term and short-term variations of the gas production rate. Indeed, we note that in addition to the study of the long term evolution of gas production in $\mathrm{P} / \mathrm{Halley}$, there were also successful programs to monitor short term variability related to gaseous outbursts (Silva and Mirabel 1988) and the 7-day periodicity in the comet's gas production observed at other wavelengths (Colom and Gérard 1988). Finally, we note that as sensitivity improves at radio wavelengths, it may be possible to investigate the magnetic field of the coma through polarimetric studies as discussed by Gérard (1985).

The access to the coma kinematics through the radio $\mathrm{OH}$ line shapes is almost unique. The systematic observation of $\mathrm{OH}$ line shape in a large sample of comets with different physical conditions will allow us to measure the coma expansion velocity and test hydrodynamical models, thereby providing us with an improved understanding of an fundamental area of cometary physics. The measurement of the coma 
velocity anisotropy will provide access to the determination of nongravitational forces and, ultimately, to the masses of comet nucleii.

\subsection{OTHER MOLECULES}

The observation of the rotational lines of $\mathrm{HCN}$ not only is interesting for cometary chemistry, but also provides a probe of cometary activity and of the coma kinematics. HCN radio lines are still difficult to observe at the present time, but technical improvements should provide access to more comets, especially through the observation of the promising $\mathrm{J}=3-2 \mathrm{HCN}$ transition.

Previous searches for cometary water at radio wavelengths have been disappointing because the rotational transitions accessible from the ground (or even from airplane altitudes) occur between high levels, which are very poorly populated in cometary atmospheres. The situation will be drastically different for transitions (such as the $1_{10^{-1}}$ transition at $557 \mathrm{GHz}$ ) between low rotational levels, which could be observed by future space submillimeter telescopes. Observation of this transition would permit studies of cometary activity and of coma kinematics as well as providing direct measurements of the temperature of the gas in the coma.

Other candidate parent molecules should be sought in bright comets in order to improve our knowledge of cometary chemistry. In fact, given the history of searches for $\mathrm{HCN}$, it is probably worthwhile to continue to search for interesting species even if they have already been sought in previous comets. For this, the access to the submillimeter range, which is often better suited for the rotational transitions of many species, will be a major improvement (Crovisier 1986). The use of interferometers such as the VLA will also continue to be very interesting for searching for short scale-lived species such as $\mathrm{H}_{2} \mathrm{CO}$.

Ions such as $\mathrm{CO}^{+}$or $\mathrm{H}_{3} \mathrm{O}^{+}$are abundant and have strong dipole moments. Therefore, they are promising species for radio investigations even though searches in $\mathrm{P} /$ Halley were negative. Radio detection of ions would yield interesting clues to the kinematics of the coma ionosphere, especially to the acceleration of ions by the solar wind.

\subsection{FUTURE THEORETICAL WORK}

Finally, we note that, as with observations at other wavelengths, the interpretation of cometary radio observations requires the suppport of theoretical calculations of many kinds in order to provide information on the composition and physical processes of comets. It is therefore necessary to pursue theoretical work on molecular excitation, collisions, coma kinematics and hydrodynamics in order to ensure that this feedback between radio observations and cometary physics continues. After all, in the final analysis, it is the ability of new techniques like radio astronomy to probe new physical processes in comets that makes this work interesting and enjoyable.

\section{References}

A'Hearn, M. F., Hoban, S., Birch, P. V., Bowers, C., Martin, R., and Klinglesmith, D. A. (1986)

Cyanogen jets in comet Halley. Nature 324, 649-651

Altenhoff, W. J., Batrla, W., Huchtmeier, W. K., Schmidt, J., Stumpff, P., and Walmsley, C. M. (1983)

Radio observations of comet 1983d. Astron. Astrophys. 125, L19-L22

Altenhoff, W. J., Huchtmeier, W. K., Schmidt, J., Schraml, J. B., Stumpff, P., and Thum, C. (1986)

Radio continuum observations of comet Halley. Astron. Astrophys. 164, 227-230

Altenhoff, W. J., Huchtmeier, W. K., Kreysa, E., Schmidt, J., Schraml, J. B., and Thum, C. (1989)

Radio continuum observations of comet Halley at $250 \mathrm{GHz}$. Astron. Astrophys. 222, 323-328. 
Alurkar, S. K., Bhonsle, R. V., and Sharma, A. K. (1986) Radio observations of PKS2314+03 during occultation by comet Halley. Nature 322, 439-441

Ananthakrishnan, S., Bhandari, S. M., and Pramesh, R. A. (1975) Occultation of radio source PKS 2025-15 by comet Kohoutek (1973f). Astrophys. Space Sci. 37, 275-282

Ananthakrishnan, S., Manoharan, P. K., and Venugopal, V. R. (1987) Occultation observations of compact radio sources through comet Halley's plasma tail. Nature 329, 698-700

Bajaja, E., Arnal, E. M., Cersosimo, J. C., Colomb, F. R., Martin, M. C., Mazzaro, J., Morras, R., Olalde, J. C., Poppel, W. G. L., Silva, A. M., Mirabel, I. F., and Boriakoff, V. (1987a) Comet Halley: OH absorption of galactic background radio sources. Astrophys. J. 313, 906-909

Bajaja, E., Morras, R., Poppel, W. G. L., Cersosimo, J. C., Martin, M. C., Mazzaro, J., Olalde, J. C., Silva, A., Arnal, M. E., Colomb, F. R., Mirabel, I. F., and Boriakoff, V. (1987b) Observation of the $\mathrm{OH} 1667 \mathrm{MHz}$ line toward comet Halley from the southern hemisphere. Astrophys. J. 322, 549-558

Baum, S., and Hoban, S. (1986) A search for the millimeter wave transitions of CO+ in comet $\mathrm{P} / \mathrm{Halley}$. Icarus 67, 515-519

Berulis, I. I., Zinchenko, I. G., Losovskii, B. Ya., Sorochenko, R. L., and Tolmachev, A. M. (1987) A search for millimeter-wave $\mathrm{H}_{2} \mathrm{O}$ and $\mathrm{HC}_{3} \mathrm{~N}$ emission from comet Halley (in Russian). Pisma Astron. Zh. 13, 526-529 (English translation: Sov. Astron. Lett. 13, 217-218)

Biraud, F., Bourgois, G., Crovisier, J., Fillit, R., Gérard, E., and Kazés, I. (1974) OH observation of comet Kohoutek (1973) at $18 \mathrm{~cm}$ wavelength. Astron. Astrophys. 34, 163-166

Bird, M.K., Huchtmeier, W. K., von Kap-herr, A., Schmidt, J., and Walmsley, C. M. (1987) Searches for parent molecules at MPIfR. in "Cometary Radio Astronomy," Proceedings of an NRAO Workshop, W.M. Irvine, F.P. Schloerb, and L.E. Tacconi-Garman (Eds.), Green Bank WV, USA. p. 85-89

Bockelée-Morvan, D. (1987) A model for the excitation of water in comets. Astron. Astrophys. 181, 169-181

Bockelée-Morvan, D., Bourgois, G., Crovisier J., and Gérard, E. (1989) Comet Okazzaki-Levy-Rudenko (1989r) IAU Circular No. 4882

Bockelée-Morvan, D., and Crovisier, J. (1985) Possible parents for the cometary CN radical: Photochemistry and excitation conditions. Astron. Astrophys. 151, 90-100

Bockelée-Morvan, D., and Crovisier, J. (1987) The role of water in the thermal balance of the coma. In "Symposium on the Diversity and Similarity of Comets", ESA SP-278, 235-240

Bockelée-Morvan, D., Crovisier, J., Baudry, A., Despois, D., Perault, M., Irvine, W. M., Schloerb, F. P., and Swade, D. (1984) Hydrogen cyanide in comets: Excitation conditions and radio observations of comet IRAS-Araki-Alcock 1983d. Astron. Astrophys. 141, 411-418

Bockelée-Morvan, D., Crovisier, J., Despois, D., Forveille, T., Gérard, E., Schraml, J., and Thum, C. (1987) Molecular observations of comets P/Giacobini-Zinner 1984e and P/Halley 1982i at millimetre wavelengths. Astron. Astrophys. 180, 253-262

Bockelée-Morvan, D., Crovisier, J., and Gérard, E. (1989) Retrieving the coma gas expansion velocity from cometary radio $\mathrm{OH}$ line profiles. In "Asteroids, Comets, Meteors III," C-I Lagerkvist, H. Rickman, B. A. Lindblad, and M. Lindgren (Eds.), Uppsala, Sweden. pp. 267-270

Bockelée-Morvan, D., and Gérard, E. (1984) Radio observations of the hydroxyl radical in comets with high spectral resolution. Kinematics and asymmetries of the $\mathrm{OH}$ coma in C/Meier (1978 XXI), C/Bradfield (1979 X), and C/Austin (1982g). Astron. Astrophys. 131, 111-122

Bystrova, N. V., Golubchin, G. S., Gosachinskii, I. V., Morozov, A. S., Nizhel'skaya, E. K., and Yudaeva, N. A. (1987) Observations of hydroxyl in comet Halley (in Russian). Pisma Astron Zh. 13, 1100-1103 (English translation: Sov. Astron. Lett. 13, 464-465)

Campbell, D. B. (1990) Radio studies: Radar. In "Comets in the Post-Halley Era," in press.

Campbell, D. B., Harmon, J. K., and Shapiro, I. I. (1989) Radar observations of comet Halley. Astrophys. J. 338, 1094-1105

Chin, G., and Weaver, H. A. (1984) Vibrational and rotational excitation of CO in comets: Nonequilibrium calculations. Astrophys. J. 285, 858-869 
Colom, P. (1989) Observations radio du radical OH dans la comete de Halley: Variations temporelles et anisotropie de la production gazeuse. Thesis, Universite Paris VII

Colom, P., Despois, D., Paubert, G., Bockelée-Morvan, D., Crovisier, J., and Gérard, E. (1989) Periodic comet Brorsen-Metcalf (1989o). IAU Circular No. 4851

Colom, P., and Gerrard, E. (1988) A search for periodicities in the $\mathrm{OH}$ radio emission of comet P/Halley (1986 III). Astron. Astrophys. 204, 327-336

Colom, P., Gérard, E., and Crovisier, J. (1990) A study of the non-gravitational forces on comet Halley nucleus from the observations of the $\mathrm{OH}$ radio lines. In "Asteroids, Comets, Meteors III," C-I Lagerkvist, H. Rickman, B. A. Lindblad, and M. Lindgren (Eds.), Uppsala, Sweden. pp. 293-296

Combes, M. et al. (1988) The 2.5 to 12 microns spectrum of comet Halley from the IKS-VEGA experiment. Icarus $76,404-436$

Combi, M. R., and Delsemme, A. H. (1980) Neutral cometary atmospheres. I. An average random walk model for photodissociation in comets. Astrophys. J. 237, 633-640

Combi, M. R., and Smyth, W. H. (1988) Monte Carlo particle-trajectory models for neutral cometary gases. I. Models and equations. Astrophys. J. 327, 1026-1043

Crifo, J. F. (1989) Collisional coma models: An unorthodox overview. Adv. Space Res. 9, (3)197-(3)211

Cordes, J., Falchi, A., Tofani, G., Lewis, B. M., and Terzian, Y. (1986) Radio OH observations of Halley's comet. In "20th ESLAB Symposium on the Exploration of Halley's Comet", ESA SP-250, Vol. 3, 113-115

Crovisier, J. (1984) The water molecule in comets: Fluorescence mechanisms and thermodynamics of the inner coma. Astron. Astrophys. 130, 361-372 (erratum 135, 197)

Crovisier, J. (1985) Searching for cometary parent molecules at radio wavelengths. Astron. J. 90, 670-674

Crovisier, J. (1986) Sub-millimetre cometary spectroscopy. In "ESA Workshop on a Space-Borne Sub-Millimetre Astronomy Mission", ESA SP-260, 57-65

Crovisier, J. (1987) Rotational and vibrational synthetic spectra of linear parent molecules in comets. Astron. Astrophys. Suppl. Series 68, 223-258

Crovisier, J. (1989) The photodissociation of water in cometary atmospheres. Astron. Astrophys. $213,459-464$

Crovisier, J., Despois, D., Bockelée-Morvan, D., Gérard, E., Johansson, L. E. B., Ekelund, L., Winnberg, A., Ge, W., Irvine, W. M., Kinzel, W. M., Schloerb, F. P., and Swade, D. A. (1990) A search for the 3-mm lines of HCN in comet Wilson 1987 VII. In "Asteroids, Comets, Meteors III," C-I Lagerkvist, H. Rickman, B. A. Lindblad, and M. Lindgren (Eds.), Uppsala, Sweden. pp.301-304

Crovisier, J., and Encrenaz, T. (1983) Infrared fluorescence of molecules in comets: The general synthetic spectrum. Astron. Astrophys. 126, 170-182

Crovisier, J., and Le Bourlot, J. (1983) Infrared and microwave fluorescence of carbon monoxyde in comets. Astron. Astrophys. 123, 61-66

Delsemme, A. H. (1982) Chemical composition of cometary nuclei. In "Comets", L L Wilkening (Ed.), The University of Arizona Press, Tucson, Arizona, pp. 85-130

Delsemme, A. H., and Miller, D. C. (1971) Physico-chemical phenomena in comets - III the continuum of comet Burnham (1960 II). Planet. Space Sci. 19, 1229-1257

de Pater, I., Palmer, P., and Snyder L. E. (1986) The brightness distribution of OH around comet Halley. Astrophys. J. 304, L33-L36

de Pater, I., Palmer, P., and Snyder, L. E. (1990) A review of radio interferometric imaging of comets. In "Comets in the Post-Halley Era," in press

de Pater, I., Wade, C. M., Houpis, H. L. F., and Palmer, P. (1985) The nondetection of continuum radiation from comet IRAS-Araki-Alcock (1983d) at 2- to 6-cm wavelengths and its implication on the icy-grain halo theory. Icarus 62, 349-359

Despois, D., Crovisier, J., Bockelée-Morvan, D., Schraml, J., Forveille, T., and Gérard, E. (1986) Observations of hydrogen cyanide in comet Halley. Astron. Astrophys. 160, L11-L12 
Despois, D., Gérard, E., Crovisier, J., and Kazés, I. (1981) The OH radical in comets: Observation and analysis of the hyperfine microwave transitions at $1667 \mathrm{MHz}$ and $1665 \mathrm{MHz}$.

Astron. Astrophys. 99, 320-340

Duncan, R. A., Norris, R. P., Forster, J. R., Stewart, R. T., and McConnell, D. (1986) Observations of $18 \mathrm{~cm} \mathrm{OH}$ emission and absorption from Halley's comet. Proc. Astron. Soc. Australia 6, 301-303

Dobrovolski, O. V. (1958) Radio Emission of comets (in Russian). Bull. Instit. Astrofiz. Akad. Nauk. Tadzh. SSR. No. 26

Elitzur, M. (1981) On pumping of the OH ground state in comets. Astrophys. J. 246, 354-359

Falchi, A., Gagliardi, L., Palagi, F., Tofani, G., and Comoretto, G. (1987) $10.7 \mathrm{GHz}$ continuum observations of comet P/Halley. Astron. Astrophys. 187, 462-464

Festou, M. C. (1981) The density distribution of neutral compounds in cometary atmospheres. I. Models and equations. Astron. Astrophys. 95, 69-79

Galt, J. (1987) Monitoring the $1.667 \mathrm{GHz}$ OH line in comet Halley. Astron. J. 93, 747-752

Gaylard, M. J. (1987) Comet Halley hydroxyl observations at 18-cm during the transit of the galactic plane and Centaurus A. In "Cometary Radio Astronomy," Proceedings of an NRAO Workshop, W. M. Irvine, F. P. Schloerb, and L. E. Tacconi-Garman (Eds.) Green Bank, WV, USA pp. 109-119

Gérard, E. (1985) An estimate of the magnetic field strength in the $\mathrm{OH}$ coma of comet Austin (1982 VI). Astron. Astrophys. 146, 1-10

Gérard, E. (1990) On the discrepancy between $\mathrm{OH}$ production rates deduced from radio and UV observations of comets. I A comparative study of radio and UV observations of P/Halley 1986 III in late November and early December 1985. Astron. Astrophys, in press.

Gérard, E., Bockelée-Morvan, D., Bourgois, G., Colom, P., and Crovisier, J. (1987) 18-cm wavelength radio monitoring of the $\mathrm{OH}$ radical in comet $\mathrm{P} / \mathrm{Halley} 1982 \mathrm{i}$. Astron. Astrophys. $187,455-461$

Gérard, E., Bockelée-Morvan, D., Bourgois, G., Colom, P., and Crovisier, J. (1988) Observations of the $\mathrm{OH}$ radio lines in comet P/Giacobini-Zinner 1985 XIII. Astron. Astrophys. Suppl. Series $74,485-495$

Gérard, E., Bockelée-Morvan, D., Bourgois, G., Colom, P., and Crovisier, J. (1989) Observations of the $\mathrm{OH}$ radio lines in comet P/Halley 1986 III. Astron. Astrophys. Suppl. Series 77, 379-410

Gibson, D. M., and Hobbs, R. W. (1981) On the microwave emission from comets. Astrophys. J. 248, 863-866. (Errata, Astrophys. J. 269, 805-806)

Goldstein, R. M., Jurgens, R. F., and Sekanina, Z. (1984) A radar study of comet IRAS-Araki-Alcock 1983d. Astron. J. 89, 1745-1754

Gulkis, S., Batelaan, P. D., Frerking, M. A., Klein, M. J., Kuiper, T. B. H., Pickett, H. M., Schaefer, M. M., Wannier, P., Bockelée-Morvan, D., Crovisier, J., Encrenaz, P. J., Zimmermann, P., and Destombes, J. L. (1989) Search for water in comet P/Halley at $380 \mathrm{GHz}$. Astron. Astrophys. 213, 465-468

Harmon, J. K., Campbell, D. B., Hine, A. A., Shapiro, I. I., and Marsden, B. G. (1989) Radar observations of comet IRAS-Araki-Alcock 1983d. Astrophys. J. 338, 1071-1093

Haser, L. (1957) Distribution d'intensite dans la tete d'une comete. Bull. Acad. Roy. Soc. Belgique (Classe des Sciences) 43, 740-750

Hoban, S., and Baum, S. (1987) A VLA search for 2-cm continuum radiation from comet Halley. Icarus $70,264-268$

Hobbs, R. W., Brandt, J. C., and Maran, S. P. (1977) Microwave continuum radiation from Comet West (1975n). Astrophys. J. 218, 573-578

Hobbs, R. W., Maran, S. P., Brandt, J. C., Webster, W. J., and Krishna Swamy, K. S. (1975) Microwave continuum radiation from comet Kohoutek 1973f: Emission from the icy-grain halo? Astrophys. J. 201, 749-755

Hodges, R. R. (1990) Monte Carlo simulation of nonadiabatic expansion in cometary atmospheres: Halley. Icarus, in press 
Huebner, W. F., Snyder, L. E., and Buhl, D. (1974) HCN radio emission from comet Kohoutek (1973f). Icarus 23, 580-584

Irvine, W. M. et al. (1984) Radioastronomical observations of comets IRAS-Araki-Alcock (1983d) and Sugano-Saiguza-Fujikawa (1983e). Icarus 60, 215-220

Irvine, W. M., Schloerb, F. P., and Tacconi-Garman, L. E. (Eds). (1987) "Cometary Radio Astronomy," Proceedings of an NRAO Workshop, Green Bank, WV, USA

Jackson, W. M., Clark, T., and Donn, B. (1976) Radio detection of $\mathrm{H}_{2} \mathrm{O}$ in comet Bradfield (1974b). In "The Study of Comets," NASA SP-393, 272-280

Jewitt, D., and Luu, J. (1989) Periodic Comet Brorsen-Metcalf (19890). IAU Circular N0, 4852

Krankowsky, D. (1990) The composition of comets. In "Comets in the Post-Halley Era," in press

Lämmerzahl, P. et al. (1987) Expansion velocity and temperatures of gas and ions measured in the coma of comet P/Halley. Astron. Astrophys. 187, 169-173

Larson, H. P., Mumma, M. J., and Weaver, H. A. (1987) Expansion velocity and temperatures of gas and ions in the coma of comet P/Halley. Astron. Astrophys. 187, 166-173

Luo, X., Zhou, T., Yao, D., Du, S., Qian, Z., Ji, D., and Zhang, W. (1988) Radio observations of the $\mathrm{OH}$ line in comet Halley. Act. Astrophys. Sin. 8, 223-229. (English translation: Chin. Astron. Astrophys. 12, 324-327)

Luo, X., Zhou, T., Yao, D., Du, S., Qian, Z., Ji, D., and Zhang, W. (1988) Radio observations of the $\mathrm{OH}$ line in comet Halley. Vistas in Astronomy 31, 161-163

Mies, F. H. (1974) Ultraviolet fluorescent pumping of $\mathrm{OH} 18$-centimeter radiation in comets. Astrophys. J. 191, L145-L148

Rickman, H. (1986) Masses and densities of comets Hallcy and Kopff. In "Comet Nucleus Sample Return", ESA SP-249, 195-205

Rickman, H. (1989) The nucleus of comet Halley: Surface structure, mean density, gas and dust density. Adv. Space Res. 2, (3)59-(3)71

Roesler, F. L., Scherb, F., Magee, K., Harlander, J., Reynolds, R. J., Yelle, R. V., Broadfoot, A. L., and Oliversen, R. J. (1986) High spectral resolution line profiles and images of comet Halley. Adv. Space Res. 5 (No 12), 279-282

Sagdeev, R. Z., Elyasberg, P. E., and Moroz, V. I. (1988) Is the nucleus of comet Halley a low density body ? Nature 331, 240-242

Scalise, E. Jr., Montero do Vale, J. L., Vila Boas, J. W. S., Abraham, Z., Botti, L. C. L., Tateyama, C. E., Cancoro, A. C. O., Kaufman, P., and Del Ciampo, L. F. (1987) Radio observations of comet Halley at 22 and $43 \mathrm{GHz}$. In "Cometary Radio Astronomy," Proceedings of an NRAO Workshop, W. M. Irvine, F. P. Schloerb, and L. E. Tacconi-Garman (Eds.), Green Bank, WV, USA, pp. 27-30

Schliecher, D. G. (1983) The fluorescence of cometary OH and CN. Ph D thesis, University of Maryland

Schleicher, D. G., and A'Hearn, M. F. (1988) The fluorescence of cometary OH. Astrophys. J. 331, 1058-1077

Schloerb, F. P. (1988) Collisional quenching of cometary emission in the 18 centimeter $\mathrm{OH}$ transitions. Astrophys. J. 332, 524-530

Schloerb, F. P., Claussen, M. J., and Tacconi-Garman, L. E. (1987) OH radio observations of comet P/Halley. Astron. Astrophys. 187, 469-474

Schloerb, F. P., and Gérard, E. (1985) Models of cometary emission in the 18-cm OH transitions: The predicted behavior of comet Halley. Astron. J. 90, 1117-1135

Schloerb, F. P., Kinzel, W. M., Swade, D. A., and Irvine, W. M. (1986) HCN production rates from comet Halley. Astrophys. J. 310, L55-L60

Schloerb, F. P., Kinzel, W. M., Swade, D. A., and Irvine, W. M. (1987) Observations of HCN in comet P/Halley. Astron. Astrophys. 187, 475-480

Silva, A. M., and Mirabel, I. F. (1988) Gaseous outbursts in comet P/Halley. A model for the dissociation of the $\mathrm{OH}$ radical. Astron. Astrophys. 201, 350-354 
Snyder, L. E. (1982) A review of radio observations of comets. Icarus 51, 1-24

Snyder, L. E. (1986) The $18 \mathrm{~cm}$ OH lines in comets: Preparation for Halley. Astron. J. 91, 163-170

Snyder, L. E., Palmer, P., and de Pater, I. (1989) Radio detection of formaldehyde emission from comet Halley. Astron. J. 97, 246-253

Snyder, L. E., Palmer, P., and Wade, C. M. (1983) An upper limit to the microwave continuum radiation from comet Austin (1982g). Astrophys. J. 269, L21-L23

Snyder, L. E., Palmer, P., and de Pater, I. (1990) Observations of formaldehyde in comet Machholz (1988j) Icarus, in press

Swade, D. A., Schloerb, F. P., Irvine, W. M., and Kinzel, W. M. (1987) Search for molecules in comet Halley at millimeter wavelengths. In "Cometary Radio Astronomy," Proceedings of an NRAO Workshop, W. M. Irvine, F. P. Schloerb, and L. E. Tacconi-Garman (Eds.), Green Bank, WV, USA, pp. 79-83

Tacconi-Garman, L. E. (1989) Ph D thesis, University of Massachusetts

Tacconi-Garman, L. E., Schloerb, F. P., and Claussen, M. J. (1990) A kinematic model for cometary comae. In "Asteroids, Comets, Meteors III," C-I Lagerkvist, H. Rickman, B. A. Lindblad, and M. Lindgren (Eds.), Uppsala, Sweden. pp.455-458

Turner, B. E. (1974) Detection of OH at 18-centimeter wavelength in comet Kohoutek (1973f). Astrophys. J. 189, L137-L139

Van Dishoeck, E. F., and Dalgarno, A. (1984) The dissociation of OH and OD in comets by solar radiation. Icarus $59,305-313$

Walmsley, C. M. (1985) The interpretation of the radio continuum emission from comet 1983d (IRAS-Araki-Alcock). Astron. Astrophys. 142, 437-440

Weaver, H. A., Mumma, M. J., and Larson, H. P. (1990) Infrared spectroscopy of cometary parent molecules. In "Comets in the Post-Halley," in press

Winnberg, A., Ekelund, L., and Ekelund, A. (1987) Detection of HCN in comet P/Halley. Astron. Astrophys. 172, 335-341

Wootten, A., Boulanger, F., Bogey, M., Combes, F., Encrenaz, P. J., Gerin, M., and Ziurys, L. (1986) A search for interstellar $\mathrm{H}_{3} \mathrm{O}^{+}$. Astron. Astrophys. 166, L15-L18 